\title{
Ellipsoidal vortices in rotating stratified fluids: beyond the quasi-geostrophic approximation
}

\author{
Yue-Kin Tsang $†$ and David G. Dritschel \\ School of Mathematics and Statistics, University of St Andrews, St Andrews KY16 9SS, UK \\ (Received ?; revised ?; accepted ?. - To be entered by editorial office)
}

We examine the basic properties and stability of isolated vortices having uniform potential vorticity in a non-hydrostatic rotating stratified fluid, under the Boussinesq approximation. For simplicity, we consider a uniform background rotation and a linear basic-state stratification for which both the Coriolis and buoyancy frequencies, $f$ and $N$, are constant. Moreover, we take $f / N \ll 1$, as typically observed in the Earth's atmosphere and oceans. In the small Rossby number 'quasigeostrophic' limit, when the flow is weak compared to the background rotation, there exists exact solutions for steadily-rotating ellipsoidal volumes of uniform potential vorticity in an unbounded flow (Zhmur \& Shchepetkin 1991; Meacham 1992). Furthermore, a wide range of these solutions are stable so long as the horizontal and vertical aspect ratios $\lambda$ and $\mu$ do not depart greatly from unity (Dritschel et al. 2005). In the present study, we examine the behaviour of ellipsoidal vortices at Rossby numbers up to near unity in magnitude. We find that there is a monotonic increase in stability as one varies the Rossby number from nearly -1 (anticyclone) to nearly +1 (cyclone). That is, quasi-geostrophic vortices are more stable than anticyclones at finite (negative) Rossby number, and generally less stable than cyclones at finite (positive) Rossby number. Ageostrophic effects strengthen both the rotation and the stratification within a cyclone, enhancing its stability. The converse is true for an anticyclone. For all Rossby numbers, stability is reinforced by increasing $\lambda$ towards unity or decreasing $\mu$. An unstable vortex often restabilises by developing a near-circular cross section, typically resulting in a roughly ellipsoidal vortex, but occasionally a binary system is formed. Throughout the nonlinear evolution of a vortex, the emission of inertiagravity waves is negligible across the entire parameter space investigated. Thus, vortices at small to moderate Rossby numbers, and any associated instabilities are (ageostrophically) balanced. A manifestation of this balance is that, at finite Rossby number, an anticyclone rotates faster than a cyclone.

Key words:

\section{Introduction}

Vortices of widely-varying sizes exist in the Earth's atmosphere and oceans, as well as in other planetary atmospheres. From synoptic-scale tropical cyclones to mesoscale Gulf Stream Rings and submesoscales eddies in the upper ocean, the structure and behaviour of these 'geophysical' vortices are strongly influenced by both the stable vertical density stratification and the planetary rotation. Vortices affect the collective motion of the atmosphere and the oceans and hence play an important role in our climate system. For example, anticyclonic mesoscale lens-shaped vortices called 'meddies' (McDowell \& Rossby 1978) found in the Gulf of Cadiz are thought to

$\dagger$ Present address: School of Mathematics, University of Edinburgh, Edinburgh EH9 3JZ, UK 
contribute significantly to the transport of heat and salinity into the North Atlantic Ocean. Geophysical vortices can often exist and evolve over long periods of time with minimal interaction with other coherent structures that may exist in the flow (Carton 2010). A primary question then is why vortices exist and persist for many internal rotation periods, even when the background flow provides continuous disturbances. To address this, a natural starting point is to consider an isolated vortex, and determine how its shape and its strength affect its stability. While this approach is simplistic, we argue that it is relevant to the way in which vortices lose stability after being critically distorted by a background flow.

The study of isolated vortical structures, vortices, dates back to Rankine and Kirchhoff in the 19th century. The circular Rankine vortex (Rankine 1858) and the elliptical Kirchhoff vortex (Kirchhoff 1876; Lamb 1932) are exact, shape-preserving solutions to the two-dimensional Euler equations for patches of uniform vorticity embedded in an unbounded potential flow. Extensions to include an ambient strain and shear flow were made by Chaplygin (Meleshko \& van Heijst 1994), Moore \& Saffman (1971) and Kida (1981), and stability was investigated by Meacham et al. (1990) and Dritschel (1990). For a review of early works on two-dimensional vortex dynamics, see Meleshko \& van Heijst (1994).

In the more general geophysical context, isolated vortices called 'rodons' (Cushman-Roisin et al. 1985; Young 1986) have been found as exact solutions to the single-layer shallow-water equations (having a variable fluid depth), a model of warm-core rings shed by the Gulf stream. Płotka \& Dritschel (2012) examined a wide class of vortex patch equilibria with uniform potential vorticity (PV) in the quasi-geostrophic (QG) shallow-water equations, determining both their equilibrium shapes and stability numerically. Płotka \& Dritschel (2014) generalised this class to finite Rossby number, i.e. to the full shallow-water equations, and introduced the concept of 'quasi-equilibria': vortex solutions which steadily rotate apart from exceptionally weak gravity wave radiation. These are almost shape-preserving.

Some generalisations to three-dimensional rotating, continuously-stratified flows were made by Zhmur \& Shchepetkin (1991) and Meacham (1992), exploiting simplifications afforded by the QG approximation. They considered ellipsoidal volumes of uniform PV embedded in an otherwise quiescent unbounded fluid. Meacham (1992) showed that such QG ellipsoidal vortices are steadily-rotating shape-preserving solutions, hereafter 'equilibria'. Moreover, Meacham (1992) found that many vortices are linearly stable if close to a spherical form in the vertically-stretched coordinate system $(x, y, N z / f)$ where $f$ and $N$ are the Coriolis and buoyancy frequencies respectively. This coordinate system is a natural one for QG flows. Using a complementary approach, Dritschel et al. (2005) found that these vortices are in fact stable over a much wider parameter range, and furthermore examined their nonlinear stability and long-time evolution.

Meacham et al. (1994) further generalised the ellipsoidal model to include a uniform background horizontal and vertical shear. Meacham et al. (1997) showed that the time-dependent vortex motion (which is potentially chaotic) can be described exactly in terms of a finite degreeof-freedom model obtained from the method of 'Hamiltonian moment reduction'. The stability of QG ellipsoidal vortices has been studied for some special background flows, e.g. a pure horizontal strain flow (Meacham et al. 1994) and a pure shear flow for which the strain rate equals the background rotation (Hashimoto et al. 1999). McKiver \& Dritschel (2006) studied the combined effects of horizontal strain and vertical shear on QG ellipsoidal vortices, analysing their linear stability to both ellipsoidal and non-ellipsoidal disturbances. Additional solutions to the QG equations representing a tilted spheroid rotating steadily about the vertical axis were discovered by Miyazaki et al. (1999), who also investigated their linear stability.

In a strongly stratified and rapidly rotating flow (having small Froude and Rossby numbers) there is a time-scale separation between the fast inertia-gravity waves (IGWs) and the slow 'balanced' vortical motions. This motivates the use of simplified balanced models — reduced models 
which filter out IGWs - for studying the slow PV-controlled dynamics (see Ford et al. (2000) and references therein). There is a direct analogue here to filtering sound waves by making the incompressible flow approximation. One of the simplest such balanced models, obtained in the limit of zero Rossby and Froude number (keeping an $\mathcal{O}(1)$ ratio), is the QG model (Charney 1948; Salmon 1998; Vallis 2006). In this model, the fluid motion and perturbation density field are entirely controlled by a single scalar quantity, the QG potential vorticity. This results in a major simplification of the original governing equations: one scalar field determines every aspect of the dynamics, the induced flow depends linearly on PV, and the system evolves at a slow rate proportional to the maximum PV, which is conserved in an adiabatic, frictionless flow. This may seem almost trivial, but what remains is nonlinear PV advection: PV induces the very flow which modifies its distribution. This can lead to complex, turbulent motion - albeit in a layerwise, two-dimensional sense.

Despite its broad applicability, the QG model is a balanced model of the lowest order and inevitably has its limitations. There are situations where departures from the QG limit are significant. For example, many mesoscale ocean eddies (Chelton et al. 2011), including cyclonic and anticyclonic rings detached from major ocean currents (Olson 1991; Lentini et al. 2006) and meddies (Prater \& Sanford 1994; Paillet et al. 2002), can have moderate Rossby numbers in the range of $0.1-1$. Submesoscale eddies in the Southern California Bight tend to be ageostrophic with $\mathcal{O}(1)$ Rossby numbers (Kim 2010). Under these conditions, it has been shown that geophysical flows spontaneously emit IGWs during their evolution (Ford et al. 2000; Viúdez 2007; Ólafsdóttir et al. 2008), indicating the nonexistence of a strictly balanced system. Moreover, the QG model fails to distinguish between cyclonic and anticyclonic motions, due to the fact that the QG flow fields are linearly related to PV.

To better understand vortex dynamics in naturally-occurring geophysical flows, there is a compelling need to go beyond our predominantly QG-based understanding. Specifically, we seek to answer key questions impacting on the validity of the QG approximation. How do ageostrophic effects change the stability and long-time evolution of a vortex? What role is played by IGWs? For how long can we expect a balanced model to faithfully represent vortex dynamics within a more complete model containing IGWs and higher-order ageostrophic effects? It is noteworthy that these questions are relevant to many practical applications in dynamical meteorology where the concept of balance is central to the understanding of large-scale vortical flows.

In this paper, we consider an incompressible stratified fluid in a three-dimensional rotating domain. For practical reasons, we are restricted to consider a finite domain, here triply-periodic. Following many previous studies and to retain some simplicity, both the Coriolis and the buoyancy frequencies $f$ and $N$ are taken to be constant. Moreover, the Boussinesq approximation is employed. However, we do not assume hydrostatic balance nor geostrophic balance.

We study arguably the simplest vortex form possible, namely an ellipsoidal volume of uniform PV (or PV anomaly relative to a constant background). While in the QG model described above there are exact solutions for steadily-rotating ellipsoidal vortices, no analogous solutions are known to exist for the non-hydrostatic Boussinesq equations. Instead we generate 'quasi-equilibria' by a novel numerical PV ramping procedure which spins up a non-hydrostatic, ageostrophic flow with minimal excitation of IGWs. The solutions thus obtained are remarkably close to an ellipsoid shape. They are not strictly in equilibrium, in any frame of reference, due to the weak emission of IGWs occurring for any non-symmetric vortex shape. However, the emission is so weak that we may regard the solutions as equilibria and sensibly investigate their stability and long-time nonlinear evolution.

The problem investigated is governed by four parameters: $f / N$, the PV-based Rossby number $q_{0}$, the initial horizontal aspect ratio $\lambda_{0}$ and the initial vertical aspect ratio $\mu_{0}$. For $f / N \ll 1$, the dependence on the first two parameters is negligible (Smyth \& McWilliams 1998; Dritschel \& 
McKiver 2014), allowing us to focus attention on the remaining three parameters. We mention that the linear stability of an axisymmetric columnar vortex in a non-hydrostatic Boussinesq fluid has been studied by Smyth \& McWilliams (1998), but this lies outside the parameter space investigated here.

In $\S 2$, the equations of motion are recast into a form suitable for flows that are close to a state of balance. We then discuss the numerical method and initialisation procedure employed. In $\S 3$, we follow the nonlinear evolution of the vortex using an equivalent oriented ellipsoid. Phase diagrams are constructed identifying the stability boundary and categorising the long-time nearequilibrium state in terms of the vortex PV anomaly or Rossby number $q_{0}$, and the initial vortex shape $\lambda_{0}$ and $\mu_{0} . \S 4$ discusses the vortex rotation rate, specifically its correlation with the vortex shape, and the asymmetric role played by ageostrophic effects on cyclones and anticyclones. $\S 5$ analyses the structure and significance of balanced and imbalanced motions. The balanced flow is separated into a QG part and a remaining, higher-order ageostrophic one, to clarify the impact of ageostrophic effects on vortex stability. The imbalanced flow is shown to take the form of radiating IGWs, albeit of very small amplitude across the entire parameter space. Finally, a few conclusions and suggestions for future study are provided in $\S 6$.

\section{Mathematical model and numerical method}

\subsection{Non-hydrostatic Boussinesq equations}

We consider a rotating incompressible fluid with velocity $\boldsymbol{u}=(u, v, w)$ under the $f$-plane approximation. With $z$ the vertical coordinate normal to the geoid, the fluid density $\rho$ and pressure $p$ are written as

$$
\begin{aligned}
& \rho(\boldsymbol{x}, t)=\rho_{0}+\bar{\rho}(z)+\rho^{\prime}(\boldsymbol{x}, t), \\
& p(\boldsymbol{x}, t)=\bar{p}(z)+p^{\prime}(\boldsymbol{x}, t),
\end{aligned}
$$

where $\rho_{0}$ is a constant reference density. The basic-state quantities $\rho_{0}+\bar{\rho}$ and $\bar{p}$ satisfy hydrostatic balance

$$
\frac{\mathrm{d} \bar{p}}{\mathrm{~d} z}=-\left(\rho_{0}+\bar{\rho}\right) g
$$

where both gravitation and centrifugal force contribute to the constant $g$ (Salmon 1998). The perturbation buoyancy $b$ and the buoyancy frequency $N$ are then defined through

$$
\begin{aligned}
b & =-\rho_{0}^{-1} g \rho^{\prime}, \\
N^{2} & =-\rho_{0}^{-1} g \frac{\mathrm{d} \bar{\rho}}{\mathrm{d} z} .
\end{aligned}
$$

Here, for simplicity, we take $N$ to be constant, corresponding to a linear basic-state density profile.

Making the Boussinesq approximation $\left(\bar{\rho}, \rho^{\prime} \ll \rho_{0}\right)$, the equations of motion may be simplified to

$$
\begin{aligned}
\frac{\mathrm{D} \boldsymbol{u}}{\mathrm{D} t}+f \hat{\boldsymbol{k}} \times \boldsymbol{u} & =-\rho_{0}^{-1} \nabla p^{\prime}+b \hat{\boldsymbol{k}}, \\
\frac{\mathrm{D} b}{\mathrm{D} t}+N^{2} w & =0, \\
\nabla \cdot \boldsymbol{u} & =0,
\end{aligned}
$$

where $\mathrm{D} / \mathrm{D} t$ is the material derivative, $\hat{\boldsymbol{k}}$ is the unit vector in the $z$-direction and $f$ is the Coriolis frequency (twice the constant background rotation rate). 


\subsection{Potential vorticity and ageostrophic vorticty}

Following Dritschel \& Viúdez (2003), we reformulate (2.6) using a set of variables that makes explicit the leading-order hydrostatic-geostrophic balance at small Rossby number. To this end, let us define the dimensionless total PV $Q$ and PV anomaly $q$ as

$$
Q=1+q=\frac{1}{f}(\boldsymbol{\omega}+f \hat{\boldsymbol{k}}) \cdot \boldsymbol{\nabla}\left(\frac{b}{N^{2}}+z\right),
$$

where the vorticity $\boldsymbol{\omega}=\left(\boldsymbol{\omega}_{\mathrm{h}}, \zeta\right)=\boldsymbol{\nabla} \times \boldsymbol{u}$. The expression in the second bracket above is the isopycnal height. It is related to the density through $b / N^{2}+z=\left(1-\rho / \rho_{0}\right) g / N^{2}$. Hence, $Q$ is essentially the component of the absolute vorticity $\boldsymbol{\omega}+f \hat{\boldsymbol{k}}$ along the density gradient, normalised by $f$. The basic state, $\boldsymbol{\omega}=b=0$, then has $Q=1$ and $q=0$.

A natural choice for the Rossby number is the PV anomaly $q$ itself, specifically its extreme value. Material conservation of PV implies that this Rossby number is constant. Its relation to the traditional Rossby number $U / f L$ may be seen by non-dimensionalising variables using QG scaling, i.e., $(x, y)=L(\tilde{x}, \tilde{y}), z=H \tilde{z}, t=(L / U) \tilde{t},(u, v)=U(\tilde{u}, \tilde{v}), w=(U / f L)(H / L) U \tilde{w}$ and $b=(U / f L) N^{2} H \tilde{b}$, where $L$ and $H$ are typical horizontal and vertical length scales respectively, and $U$ is a typical horizontal velocity scale. Then the PV may be written

$$
Q=1+\frac{U}{f L}\left(\frac{\partial \tilde{b}}{\partial \tilde{z}}+\tilde{\zeta}\right)+\left(\frac{U}{f L}\right)^{2} \tilde{\boldsymbol{\omega}} \cdot \tilde{\nabla} \tilde{b}
$$

The non-dimensional variables, with tildes, are of $\mathcal{O}(1)$. Hence, to leading order the PV anomaly $q$ is just the traditional Rossby number. The extreme value of $q$ is a better choice as it does not require ad hoc choices for $U$ and $L$.

The PV alone however is not sufficient to describe the dynamics of the flow, since the original system (2.6) has three independent time derivatives, after taking into account incompressibility. Motivated by QG balance, two additional variables are chosen from the horizontal (vector) component of the dimensionless ageostrophic (and non-hydrostatic) vorticity

$$
\boldsymbol{A} \equiv \frac{\omega}{f}+\frac{\nabla b}{f^{2}}
$$

Notably, the horizontal component $\boldsymbol{A}_{\mathrm{h}}=0$ for a flow in thermal wind balance (apart from often negligible contributions from horizontal derivatives of the vertical velocity). Therefore, $\boldsymbol{A}_{\mathrm{h}}$ captures higher-order ageostrophic motions beyond the leading-order hydrostatic-geostrophic balance. These motions include inertia-gravity waves, but are typically dominated by higherorder balance effects (Dritschel \& Viúdez 2007).

\subsection{Reformulation of the governing equations}

Instead of $\{\boldsymbol{u}, b, p\}$, we shall use $\left\{q, \boldsymbol{A}_{\mathrm{h}}\right\}$ as the prognostic variables to explicitly enforce material conservation of PV. This enables a more accurate representation of both the balanced, PV-controlled dynamics as well as the residual imbalance (Dritschel \& Viúdez 2003, 2007). The equations of motion for $q$ and $\boldsymbol{A}_{\mathrm{h}}$ are

$$
\begin{gathered}
\frac{\mathrm{D} q}{\mathrm{D} t}=0, \\
\frac{\mathrm{D} \boldsymbol{A}_{\mathrm{h}}}{\mathrm{D} t}+f \hat{\boldsymbol{k}} \times \boldsymbol{A}_{\mathrm{h}}=\frac{1}{f}(\boldsymbol{\omega} \cdot \boldsymbol{\nabla}) \boldsymbol{u}_{\mathrm{h}}+\left(1-\sigma^{-2}\right) \nabla_{\mathrm{h}} w-\frac{1}{f^{2}}\left(\nabla_{\mathrm{h}} \boldsymbol{u}\right) \cdot \boldsymbol{\nabla} b
\end{gathered}
$$


where $\boldsymbol{u}_{\mathrm{h}}$ is the horizontal velocity, $\boldsymbol{\nabla}_{\mathrm{h}}$ is the horizontal gradient operator and the dimensionless parameter

$$
\sigma \equiv \frac{f}{N}
$$

We set $\sigma=0.1$ (a typical value for the oceans) for all the results reported here. The results are not sensitive to this parameter so long as $\sigma \lesssim 1$ (Dritschel \& McKiver 2014).

The fields $\boldsymbol{u}$ and $b$ can be recovered from $\left\{q, \boldsymbol{A}_{\mathrm{h}}\right\}$ as follows. Expressing $\boldsymbol{A}$ in terms of a potential $\varphi$,

$$
\boldsymbol{A}=\nabla^{2} \varphi
$$

we have from (2.9),

$$
\begin{gathered}
\boldsymbol{u}=-f \boldsymbol{\nabla} \times \boldsymbol{\varphi}, \\
b=f^{2} \boldsymbol{\nabla} \cdot \boldsymbol{\varphi} .
\end{gathered}
$$

The horizontal (vector) component $\varphi_{\mathrm{h}}$ and the vertical component $\phi$ of the potential $\varphi$ are obtained from $\left\{q, \boldsymbol{A}_{\mathrm{h}}\right\}$ by inverting the following relations,

$$
\begin{gathered}
\boldsymbol{A}_{\mathrm{h}}=\nabla^{2} \boldsymbol{\varphi}_{\mathrm{h}}, \\
q=\left(\nabla_{\mathrm{h}}^{2}+\sigma^{2} \frac{\partial^{2}}{\partial z^{2}}\right) \phi-\left(1-\sigma^{2}\right) \nabla_{\mathrm{h}} \cdot \frac{\partial \boldsymbol{\varphi}_{\mathrm{h}}}{\partial z}+\sigma^{2} \boldsymbol{\nabla}(\boldsymbol{\nabla} \cdot \boldsymbol{\varphi}) \cdot\left[\nabla^{2} \boldsymbol{\varphi}-\boldsymbol{\nabla}(\boldsymbol{\nabla} \cdot \boldsymbol{\varphi})\right] .
\end{gathered}
$$

Once $\varphi_{\mathrm{h}}$ is known by solving the linear elliptic equation (2.15), we can rewrite (2.16) as a double Monge-Ampère equation in the unknown $\phi$,

$$
\sigma^{2}\left(\phi_{z z} \nabla_{\mathrm{h}}^{2} \phi-\phi_{x z}^{2}-\phi_{y z}^{2}\right)+B_{1} \nabla_{\mathrm{h}}^{2} \phi+B_{2} \phi_{z z}+C_{1} \phi_{x z}+C_{2} \phi_{y z}+D=0
$$

with the coefficients $B_{1}, B_{2}, C_{1}, C_{2}$ and $D$ depending on $\sigma, \varphi_{\mathrm{h}}$ and $q$.

\subsection{The CASL algorithm and near-balanced initialisation}

We solve the prognostic equations (2.10) numerically in a triply-periodic box of horizontal side lengths $2 \pi L \sigma^{-1} \times 2 \pi L \sigma^{-1}$ and height $2 \pi L$. In the following, we scale length and time such that $L=1$ and $N=2 \pi$. Hence, the bouyancy period is 1 and (given $\sigma=0.1$ ) the inertial period $T_{\text {ip }}=2 \pi / f=10$.

The PV anomaly $q$ is represented by contours on isopycnal surfaces. The evolution of $q$, governed by $(2.10 a)$, is carried out by solving $\mathrm{d} x / \mathrm{d} t=u(\boldsymbol{x}, t)$ and $\mathrm{d} y / \mathrm{d} t=v(\boldsymbol{x}, t)$ on these surfaces, using the Contour-Advective Semi-Lagrangian (CASL) algorithm (Dritschel \& Ambaum 1997). The ageostrophic field $\boldsymbol{A}_{\mathrm{h}}$ is discretised on a three-dimensional grid and (2.10b) is solved spectrally with explicit leap-frog time-stepping, together with very weak hyperviscosity and the high-order Fourier filtering scheme described in Hou \& Li (2007). A detailed description of the numerical method can be found in the Appendix of Dritschel \& Viúdez (2003). For the present work, we use 512 isopycnal surfaces and a grid size of $128^{3}$, apart from a few exceptional cases at double resolution noted below. The fixed time step ranges from 0.025 to 0.1 depending on $q$. For all other CASL parameters such as the surgical scale, we use the standardised values given in the bottom part of Table 1 in Fontane \& Dritschel (2009).

The initial conditions are prepared so that at time $t=0$, an ellipsoidal volume of constant PV anomaly $q_{0}$ in a near balanced state is located at the domain centre. This ensures the initial emission of inertia-gravity waves by adjustment is minimal. We achieve this by starting from a rest state at $t=-T_{\text {init }}$, then smoothly ramp up $q$ inside the ellipsoid from 0 to $q_{0}$ according to the formula

$$
q(t)=q_{0} \sin ^{2}\left[\frac{\pi}{2}\left(1+\frac{t}{T_{\text {init }}}\right)\right]
$$


while integrating the system forward over the initialisation period $-T_{\text {init }}<t<0$. We take $T_{\text {init }}=20\left|q_{0}\right| T_{\mathrm{ip}}$ to ensure minimal excitation of IGWs. Note that both the PV contours and the ageostrophic field $\boldsymbol{A}_{\mathrm{h}}$ evolve during the initialisation period. We start at $t=-T_{\text {init }}$ with an upright ellipsoid whose shape is specified by the two horizontal semi-axis lengths $a_{0}$ and $b_{0}$ $\left(a_{0}<b_{0}\right)$ and the vertical semi-axis length $c_{0}$. We define the initial horizontal aspect ratio $\lambda_{0}$ and initial vertical aspect ratio $\mu_{0}$ at $t=-T_{\text {init }}$ as follows,

$$
\begin{aligned}
\lambda_{0} & =\frac{a_{0}}{b_{0}}, \\
\mu_{0} & =\frac{c_{0}}{\sigma \sqrt{a_{0} b_{0}}} .
\end{aligned}
$$

Throughout any given simulation, the linear dimensions of the vortex in the $x-, y$ - and $z$ directions remain less than one-third of the respective domain edge lengths, so that the effects of periodicity are small. The simulations run until the vortex reaches an 'equilibrium' state, by which we mean the various vortex properties become approximately statistically stationary. The total simulated time ranges from $300 T_{\mathrm{ip}}$ to $2000 T_{\mathrm{ip}}$. With all other parameters held fixed, each simulation is characterised by $\lambda_{0}, \mu_{0}$ and the Rossby number $q_{0}$. Our principal data set consists of 308 cases spanning a large range of aspect ratios and includes both cyclones and anticyclones with $\left|q_{0}\right| \leqslant 0.5$. We have also performed a number of simulations at larger $\left|q_{0}\right|$ and higher resolution; these results will be included in some of the analyses presented in the following sections.

\section{Stability and nonlinear evolution}

We first study the stability of a given vortex and the evolution of its geometry by fitting the vortex to an equivalent oriented ellipsoid using a set of spatial moments up to second degree. The mathematical details are given in Appendix A. To measure how close the vortex is to the equivalent ellipsoid, we use the normalised overlap volume

$$
\varrho=\frac{\text { volume of the overlap region between the equivalent ellipsoid and the vortex }}{\text { volume of the vortex }} .
$$

Since by construction the equivalent ellipsoid has the same volume as the vortex, we have $0 \leqslant$ $\varrho \leqslant 1$ with $\varrho=1$ indicating the vortex is a perfect ellipsoid. Figure 1(a) exhibits a histogram of $\langle\varrho\rangle$ based on all the principal cases studied in this paper (here $\langle\cdot\rangle$ denotes a time average over the statistically steady state). Notice that $\langle\varrho\rangle \geqslant 0.9$ for all cases, indicating any departure from the ellipsoidal shape is small. As a result, the semi-axis lengths of the equivalent ellipsoid are good measures of the extent of the vortex along the three corresponding perpendicular directions.

It is not always straightforward to define the orientation of a geometrical shape (Žunić et al. 2006). Here however we find it sufficient to define the orientation of the vortex by that of the equivalent ellipsoid. For the three principal axes of the equivalent ellipsoid, we label the one with the largest projection on the $z$-axis as the vertical axis and denote its semi-length as $c(t)$. The remaining two principal axes are then the horizontal minor axis, of semi-length $a(t)$, and the horizontal major axis, whose semi-length is $b(t)$ (but see $\S 3.1$ for special cases when the ellipsoid is tumbling). The orientation of the vortex can be inferred from the tilt angle $\theta(t)$, defined as the angle between the vertical axis of the equivalent ellipsoid and the $z$-axis. For example, using a case with the smallest $\langle\varrho\rangle$, figure $1(b)$ demonstrates that the geometry of the vortex can be effectively captured by the equivalent ellipsoid. 
(a)

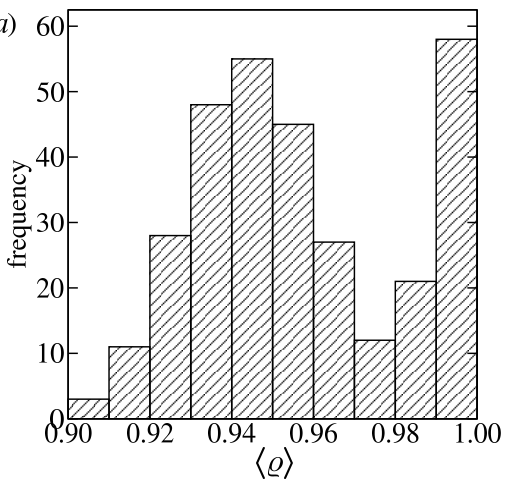

(b)

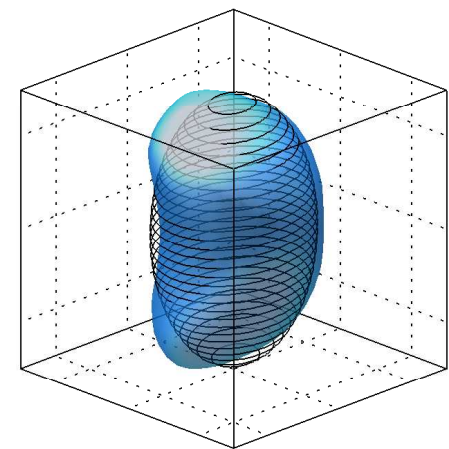

FIGURE 1. (a) Histogram of the normalised overlap volume $\langle\varrho\rangle$ defined in (3.1). A vortex with a nearly ellipsoidal shape has $\langle\varrho\rangle$ close to unity. The quasi-stable cases contribute to the spike at $\langle\varrho\rangle=1$. (b) The vortex (smooth surface) and its equivalent oriented ellipsoid (solid lines) for $q_{0}=0.25, \lambda_{0}=0.2$ and $\mu_{0}=1.8$ at a late time. The value of $\langle\varrho\rangle=0.9$ for this case is one of the smallest; nevertheless the equivalent ellipsoid manages to capture the aspect ratios and the orientation of the vortex.

\subsection{Evolution and long-term fate}

Depending on the initial conditions $\left(q_{0}, \lambda_{0}\right.$ and $\left.\mu_{0}\right)$, the vortex displays a rich variety of evolutionary behaviours and reaches different equilibrium configurations at large times. We now give an account of the various phenomena observed in the broad range of numerical experiments conducted.

1. Quasi-stable: A quasi-stable vortex deforms extremely slowly and remains close to the initial configuration during the whole simulation. Typically, the fractional change in the semiaxis lengths over $100 T_{\mathrm{ip}}$ is less than $1 \%$. A quasi-stable vortex often has $\lambda_{0} \geqslant 0.5$.

2. Shape alteration: An unstable vortex usually evolves and restablises into a new equilibrium state by changing its shape. We classify the eventual vortex shape as roughly ellipsoidal if $\langle\varrho\rangle\rangle$ 0.95 and non-ellipsoidal if $\langle\varrho\rangle \leqslant 0.95$. The evolution process often involves the shedding of vortex filaments (though there are exceptions, see $\S 3.2$ ). We find that the different scenarios for the nonlinear evolution of a QG vortex described in Dritschel et al. (2005) also apply to our non-hydrostatic Boussinesq system. Figure 2(a) shows an example of filaments being spun off and forming rings near the top and bottom of a prolate vortex $\left(\mu_{0}>1\right)$.

3. Binary system: It is not uncommon for ejected filaments to roll up into small satellite vortices which orbit around the main vortex, e.g. see figure $2(b)$. However, in a few rare cases involving a strongly horizontally-elongated vortex, the vortex develops a dumbbell shape before breaking up into a roughly ellipsoidal primary vortex and a smaller secondary vortex. The two vortices revolve around each other forming a binary system. Figure 2(c) shows an example of an anticyclonic binary system. In the analysis presented below, we focus on the primary vortex only. The formation of a binary system has not been observed in QG dynamics.

4. Shape oscillation: As a vortex becomes unstable, instead of converging towards an equilibrium shape, its boundary may start to morph away from and then back to its initial shape periodically. There are also cases where the vortex evolves away from its initial shape and oscillates between two new shapes. Furthermore, it is possible for a vortex to exhibit transient damped shape oscillations as it evolves towards an equilibrium configuration. Two modes of shape oscillation have been observed; they are illustrated in figure 2(d). Figure 2(e) shows a vortex undergoing transient shape oscillation. 
(a) Roughly ellipsoidal: $q_{0}=0.5 \lambda_{0}=0.6 \mu_{0}=2.4$
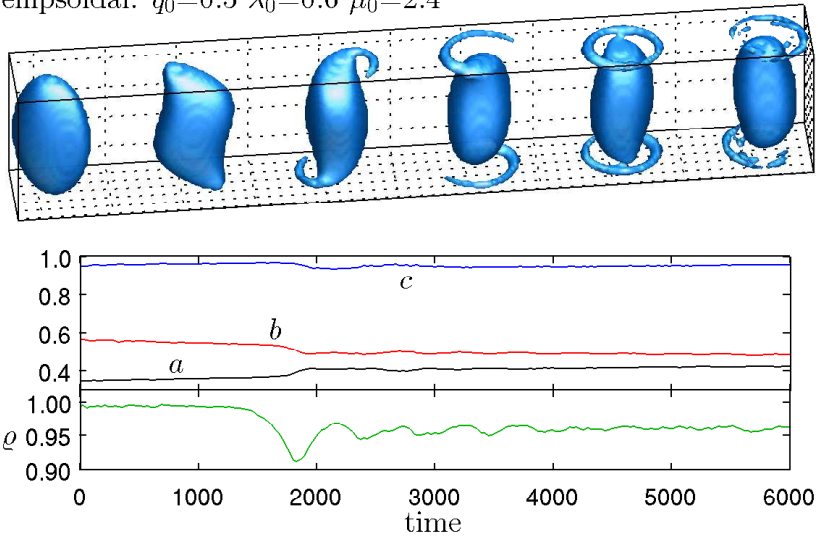

(b) Main vortex with satellites: $q_{0}-0.5 \lambda_{0}-0.3 \mu_{0}-0.4$

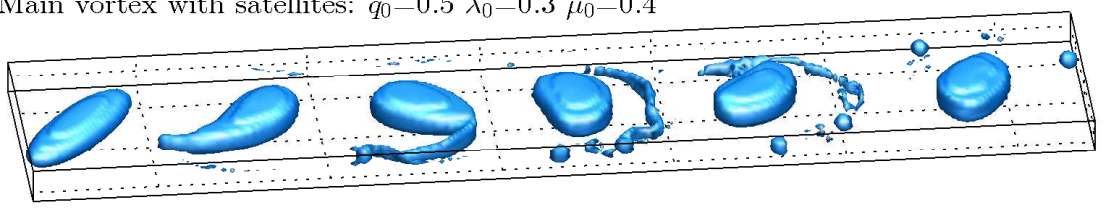

(c) Binary system: $q_{0}=-0.25 \lambda_{0}=0.2 \mu_{0}=0.6$

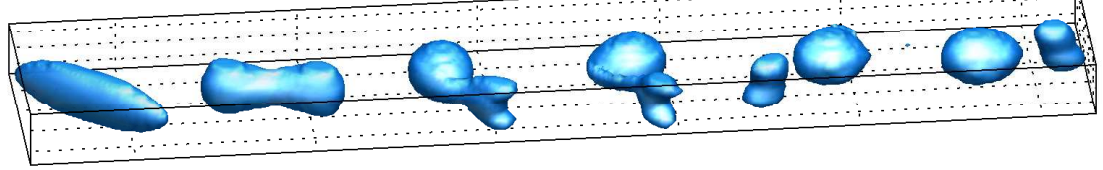

(d) Shape oscillation:
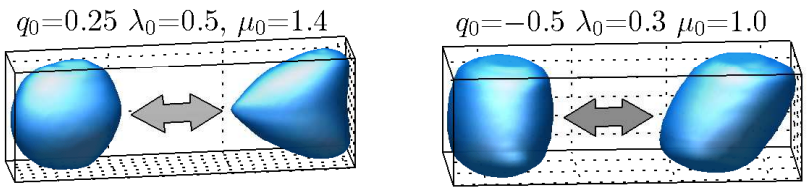

(e) Transient oscillation: $q_{0}=0.25 \lambda_{0}=0.6 \mu_{0}=2.4$
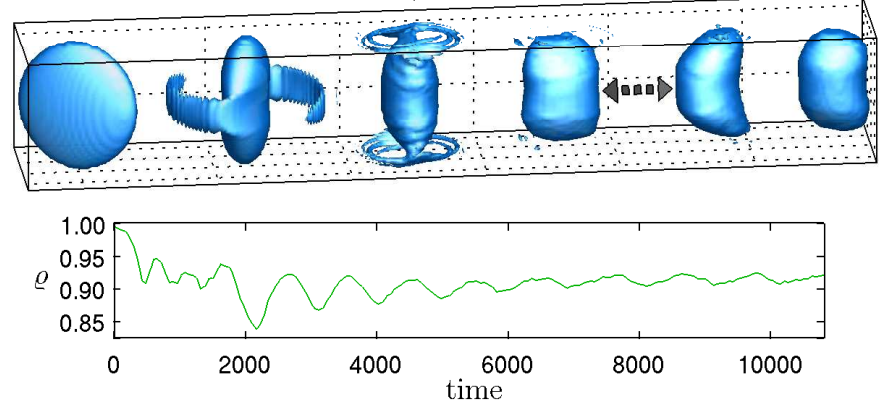

FIGURE 2. Examples of vortex evolution at different initial horizontal $\left(\lambda_{0}\right)$ and vertical $\left(\mu_{0}\right)$ aspect ratios. (a) A prolate vortex becomes unstable at $t \approx 1500$, spinning off filaments that form rings near the top and bottom of the vortex. Time series of the semi-axis lengths $a$ and $b$ of its equivalent oriented ellipsoid shows that at the time it becomes unstable, the vortex acquires a near-circular cross section $(\langle\lambda\rangle=0.87)$. The final equilibrium shape is roughly ellipsoidal as $\varrho>0.95$ at large $t$. (b) PV fragments coming off one end of a long oblate vortex as it rotates. Two major fragments form satellite vortices orbiting around the main vortex while the finer ones are extended into thin filaments. $(c)$ A binary system formed from a highly horizontally-elongated vortex. $(d)$ Two modes of vortex shape oscillation: top and bottom of the vortex moving in phase (left) or out of phase (right). (e) A vortex oscillates between two different shapes after initial strong filament shedding. The oscillation fades over time as the vortex tends to an equilibrium non-ellipsoidal shape $(\langle\varrho\rangle \approx 0.91)$. This is reflected in the damped oscillation of $\varrho(t)$ starting at $t \approx 1800$. 
5. Tumbling: We find that in some cases where $\mu_{0}$ is close to unity, the vortex starts to tumble, irrespective of any subsequent development in the vortex shape. The notion of a vertical axis, and hence the definition of the semi-axis lengths, becomes ambiguous for a tumbling ellipsoid. In such cases, after the vortex has reached a statistical steady state, we define $a(t), b(t)$ and $c(t)$ such that they have the same order in length as $a_{0}, b_{0}$ and $c_{0}$.

\subsection{Stability and phase diagram}

In $\S 3.1$ above, we described a rich variety of possible behaviours of a vortex and illustrated them in a few examples. We now present results from our full principal data set consisting of 308 simulations. We consider four different values of Rossby number: $q_{0}= \pm 0.25$ and \pm 0.5 . For the aspect ratios, $\lambda_{0}$ varies from 0.2 to 0.8 in steps of 0.1 and $\mu_{0}$ from 0.4 to 2.4 in steps of 0.2 . Figure 3 summarises the equilibrium configuration for all cases. For completeness, we also performed simulations of the QG system (Dritschel et al. 2005) representing the limit of zero Rossby number. The results are included in figure 3.

We first investigate the relation between the stability and the geometry of the vortex. Figure 3 shows that, generally, a small vertical aspect ratio and a near-circular horizontal cross section are favourable conditions for stability. In figure 3 , the circles occupy a region in the lower right of the $\lambda_{0}-\mu_{0}$ phase plane. Because the circles indicate that the vortex maintains or oscillates about its initial shape, and in anticipation of the results in $\S 3.3$ below, we loosely refer to this region as the 'stability region'. As we move away from the stability region by decreasing $\lambda_{0}$ or increasing $\mu_{0}$, we first encounter cases of unstable vortices which evolve into a roughly ellipsoidal equilibrium shape. This is then followed by the non-ellipsoidal cases as we move further from the stability region. This pattern is especially conspicuous for the QG system. For $q_{0}=-0.5$, no stability region is found within the parameter ranges considered. However, in the region below the dashed line in figure 3 , the vortex deforms noticeably over several hundred inertial periods in a smooth manner, without shedding filaments. Thus, the evolution in these cases is not unlike that of a quasi-stable vortex except that it happens over a much shorter time scale.

We now turn to the effects of the Rossby number $q_{0}$ on the stability of the vortex. From figure 3 , we see that as $q_{0}$ decreases, the stability region diminishes while its boundary shifts downwards and towards the right. Therefore we conclude that, for fixed aspect ratios $\lambda_{0}$ and $\mu_{0}$, increasing $q_{0}$ enhances stability. As a consequence, a cyclone $\left(q_{0}>0\right)$ tends to be more stable than an anticyclone $\left(q_{0}<0\right)$ of the same shape and $\left|q_{0}\right|$.

In most cases, the vortex rotates with a fixed orientation about the vertical axis (the vortex may be tilted). However, the orientation of the vortex varies if it is tumbling or undergoing the out-of-phase type shape oscillation depicted in figure $2(d)$. An interesting observation from our simulations is that a vortex with a vertical aspect ratio $\mu_{0}$ around unity is most susceptible to orientation variation (shaded symbols in figure 3).

\subsection{Aspect ratios of the late-time equilibria}

In $\S 3.2$ above, we have seen that the stability of a vortex depends crucially on its aspect ratios $\lambda$ and $\mu$. In the present section, we look into how $\lambda$ and $\mu$ change as an unstable vortex evolves into an equilibrium configuration. The discussion surrounding (3.1) points out that the equivalent oriented ellipsoid effectively captures the vortex shape. Hence, we can reliably estimate the aspect ratios $\lambda(t)$ and $\mu(t)$ of the vortex at time $t$ from $a(t), b(t)$ and $c(t)$ using expressions analogous to (2.19). The aspect ratios of the equilibrium vortex shape are then represented by the time-averaged values $\langle\lambda\rangle$ and $\langle\mu\rangle$ over the statistically steady state.

Figure 4 illustrates the change in $\lambda$ and $\mu$ between the initial shape at $t=0$ and the equi- 

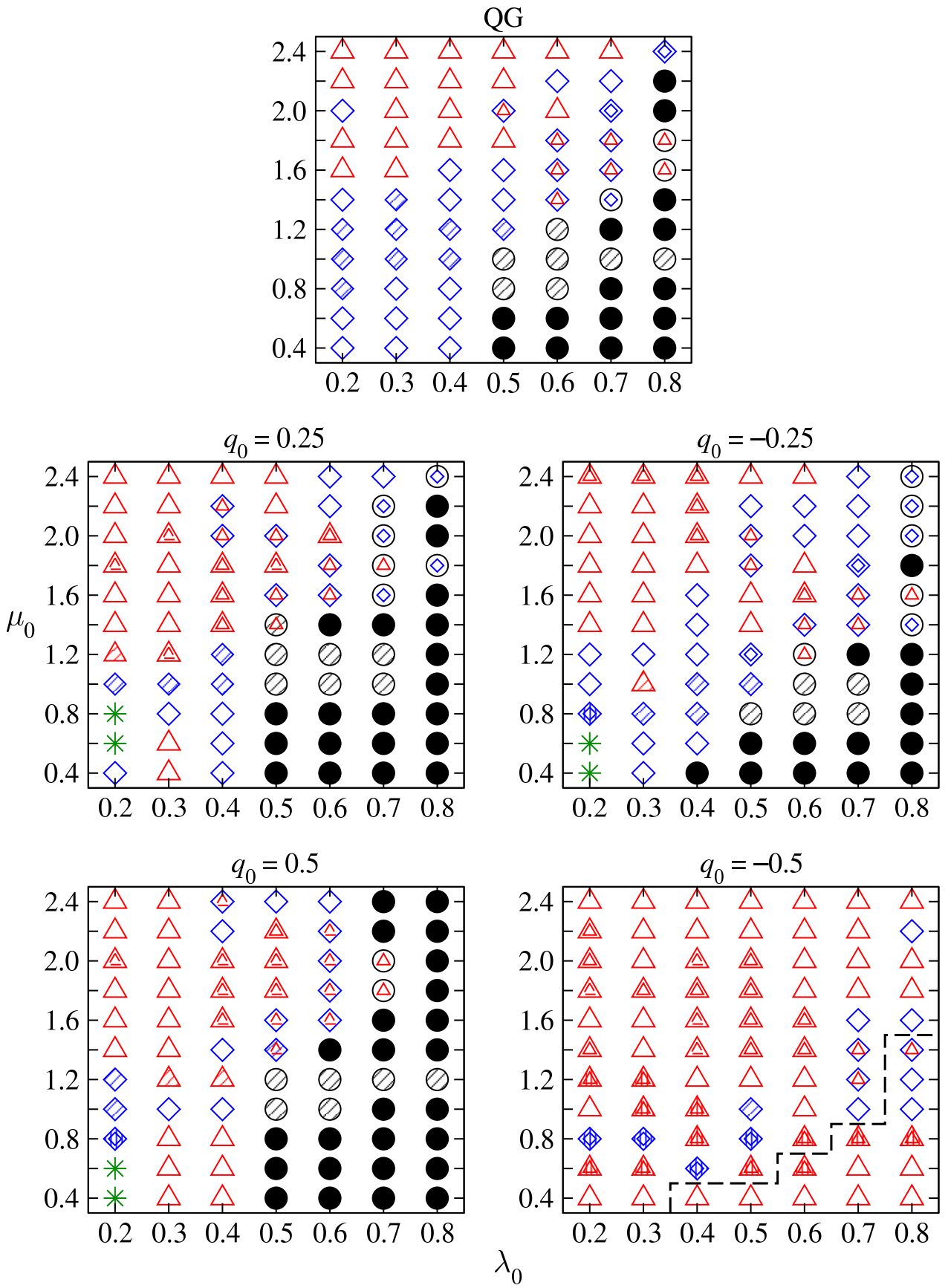

FIGURE 3. Phase diagrams showing the equilibrium configuration of a vortex at large time, for varying aspect ratios $\lambda_{0}$ and $\mu_{0}$ and for different PV anomalies or Rossby numbers $q_{0}$. The symbols denote the shape of the vortex, as follows. Circles $(\bigcirc)$ : little change from the initial shape at $t=0$; Diamonds $(\diamond)$ : roughly ellipsoidal $(\langle\varrho\rangle>0.95)$; Triangles $(\triangle)$ : non-ellipsoidal $(\langle\varrho\rangle \leqslant 0.95)$; Stars $(*)$ : binary system. The quasi-stable cases are shown with filled circles. An inscribed symbol indicates the vortex undergoes shape oscillation, while a dashed inscribed symbol means the oscillation is transient. A shaded symbol shows the orientation of the vortex is varying due to tumbling (slanted lines) or shape oscillation (vertical lines). For $q_{0}=-0.5$, inside the lower right region bounded by the dashed line are cases in which the vortex deforms smoothly without filament shedding. 

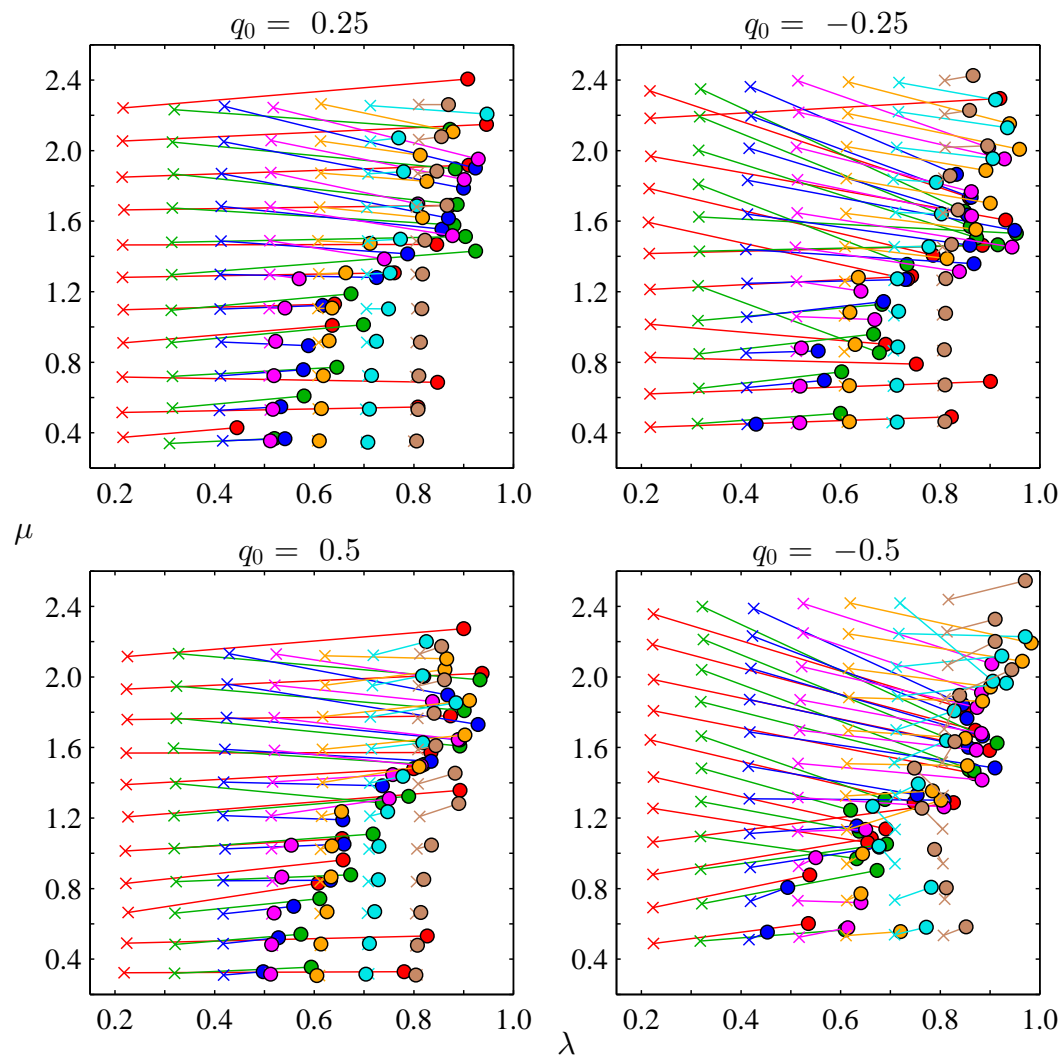

FIGURE 4. Changes in the horizontal aspect ratio $\lambda$ and the vertical aspect ratio $\mu$ of the vortex. Crosses are values at the end of the initialisation period $t=0$. Solid circles are time-averaged values $\langle\lambda\rangle$ and $\langle\mu\rangle$ over the statistically steady state at large time.

librium shape at large $t \dagger$. A prominent feature of figure 4 is that $\lambda$ and $\mu$ migrate towards and cluster around the stability boundary (except for the binary systems which evolve deep into the stability region, see $\S 3.1$ for a definition of the 'stability region'). The deformation of cyclones is mostly achieved with only a minor change in $\mu$ while a few of the anticyclones show relatively large changes in $\mu$. In the majority of cases, the vortex eventually acquires a near-circular horizontal cross section with $\langle\lambda\rangle>0.8$. The time series in figure 2(a) shows an example of $a$ and $b$ approaching each other.

We emphasize that, with the exception of the quasi-stable cases, the final equilibrium shape of the vortex is not a perfect ellipsoid, see figure 1. Thus, the clustering of $\langle\lambda\rangle$ and $\langle\mu\rangle$ seen in figure 4 suggests that the stability of the vortex depends primarily on the aspect ratios gross measures of the vortex shape - not on the detailed vortex shape. The pattern displayed in figure 4 has interesting implications for the vortex rotation rate, as elaborated in $\S 4$.

\subsection{Discussion}

In naturally-occurring geophysical flows, the process of vortex formation is likely to be highly varied and complex. Therefore, one may expect to observe vortices with a wide range of shapes. However, observed ocean eddies generally appear to have roughly circular horizontal cross sec-

$\dagger$ Recall that $\left\{\lambda\left(-T_{\text {init }}\right), \mu\left(-T_{\text {init }}\right)\right\}=\left\{\lambda_{0}, \mu_{0}\right\}$ and note there is a small difference between $\{\lambda(0), \mu(0)\}$ and $\left\{\lambda_{0}, \mu_{0}\right\}$ because the vortex is allowed to evolve during the initialisation period. 
tions. This has motivated much previous work on vortex stability in various idealised systems and has led to the idea that vortices which are highly elongated at birth are unstable and quickly evolve into more stable configurations; see for example Cushman-Roisin (1986) and Carton (2001) and references therein.

For the ellipsoidal vortices studied in the present work, we find that - in the vast majority of cases - instability results in a transition to another roughly ellipsoidal vortex, always with a more circular horizontal cross section and often with a scaled vertical aspect ratio $\mu$ closer to unity (see figure 4). A key result of the present investigation is the identification of these quasistable vortex structures emerging as the end states of evolution starting from a wide range of initial shapes, i.e. the identification of the 'stability region' mapped out by the circles in figure 4.

The form of the stability region constrains the possible geometry of a stable vortex. This leads to some interesting consequences. As just remarked, all unstable vortices restabilise by increasing their horizontal aspect ratio $\lambda$ toward unity. At equilibrium, we find $\lambda \gtrsim 0.5$. Furthermore, the most probable value for $\lambda$ is around 0.8 . This echoes what has been reported in many observations of ocean eddies (Paillet et al. 2002; Lentini et al. 2006; Kim 2010; Park et al. 2012). Also, tall vortices (large $\mu$ ) are restricted to have $\lambda \approx 1$ while flat vortices (small $\mu$ ) can exhibit a wider range of $\lambda$. Finally, since the stability boundary migrates towards smaller $\lambda$ as $q_{0}$ increases, stable highly-eccentric vortices (with small $\lambda$ ) are most likely to be strongly ageostrophic cyclones (with $q_{0}$ near unity). On the other hand, strongly ageostrophic anticyclones are restricted to have nearly circular horizontal cross section. In light of the present results, it would be worthwhile examining observational data to see where ocean eddies lie in figure 4.

As regards ageostrophic effects on stability (in the sense explained in $\S 3.1$ ), we find robustly that vortices are increasingly stable (over a broader range of initial shapes) as the signed Rossby number $q_{0}$ increases. While our analysis has largely been restricted to the range $-0.5 \leqslant q_{0} \leqslant 0.5$, a few additional simulations at $q_{0}=0.75$ suggest this trend applies over a wider range of $q_{0}$. We cannot examine $\left|q_{0}\right| \gtrsim 1$ with the PV conserving numerical algorithm employed here because then we cannot invert the PV (Dritschel \& Viúdez 2003).

\section{Vortex rotation rate}

In the majority of cases considered here, the vortex shape, specifically the boundary of the region of nonzero PV anomaly, rotates about the vertical axis at an approximately constant angular velocity $\Omega$ after the system has reached a statistically steady state. In the exceptional cases when the vortex shape oscillates or the vortex is tumbling, the magnitude or the direction of the angular velocity - or both - vary with time. For all cases, we compute the vortex rotation rate $\Omega(t)$ by tracking the projection of the horizontal major axis (defined in $\S 3$ ) of the equivalent ellipsoid on the $x-y$ plane. Figure 5 shows $\Omega(t)$ for a few selected cases, illustrating a sample of the varied behaviours that are possible. Here, we examine the late-time average rotation rate $\langle\Omega\rangle$ of the equilibrium state, in particular its dependence on $\langle\lambda\rangle$ and $\langle\mu\rangle$ and its deviation from the rotation rate $\Omega_{\mathrm{QG}}$ of a $\mathrm{QG}$ ellipsoid (Meacham 1992; Dritschel et al. 2005):

$$
\Omega_{\mathrm{QG}}(\lambda, \mu)=\left(q_{0} f\right) \mu \frac{\lambda^{-1} R_{D}\left(\mu^{2}, \lambda, \lambda^{-1}\right)-\lambda R_{D}\left(\mu^{2}, \lambda^{-1}, \lambda\right)}{3\left(\lambda^{-1}-\lambda\right)},
$$

where

$$
R_{D}(x, y, z)=\frac{3}{2} \int_{0}^{\infty} \frac{\mathrm{d} t}{\sqrt{(t+x)(t+y)(t+z)^{3}}}
$$

is Carlson's symmetric elliptic integral of the second kind (Carlson \& Gustafson 1994). Our main findings are summarised in Figure 6(a), presenting a scatter plot of $\langle\Omega\rangle$ versus $\langle\mu\rangle$ for all 


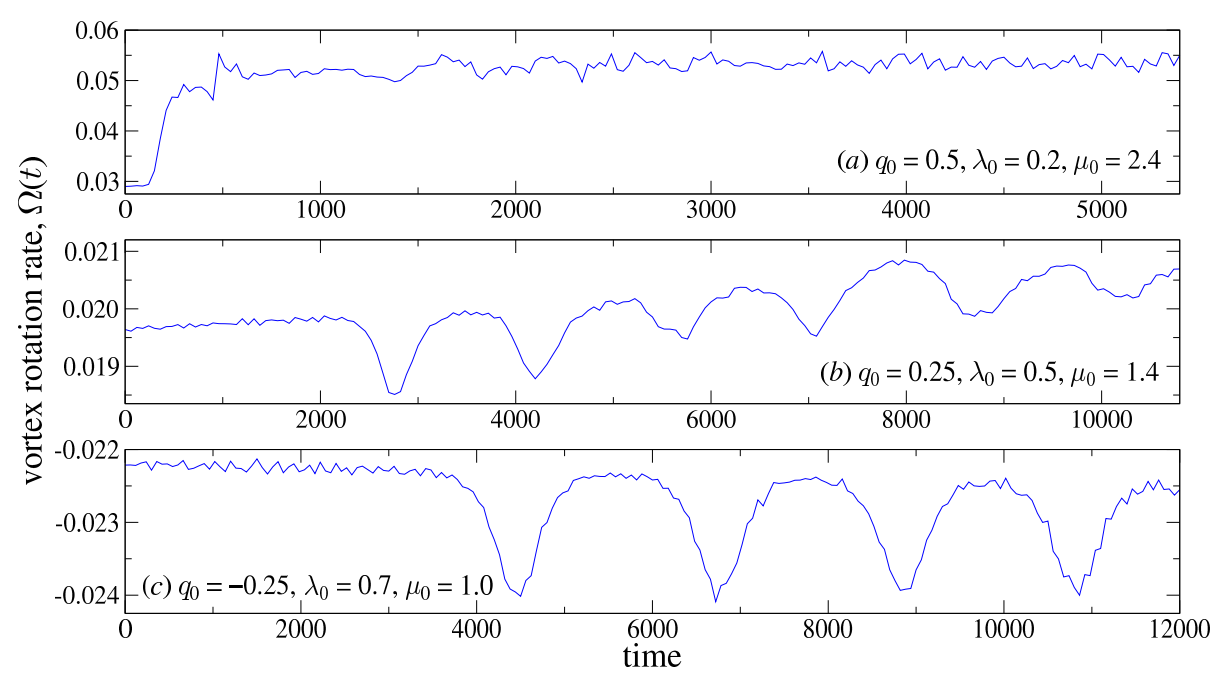

FIGURE 5. Rotation rate $\Omega(t)$ for a vortex which $(a)$ rotates approximately steadily at late times $(\langle\lambda\rangle=0.90$ and $\langle\mu\rangle=2.27$ for this case); $(b)$ exhibits shape oscillations as shown in figure $2(d)$ (the period is $\approx 150 T_{\mathrm{ip}}$; note $\Omega(t)$ decreases in the phase of the oscillation when the vortex is extending horizontally); $(c)$ starts to tumble after a period of steady rotation; the vortex maintains its initial ellipsoidal shape throughout the simulation.
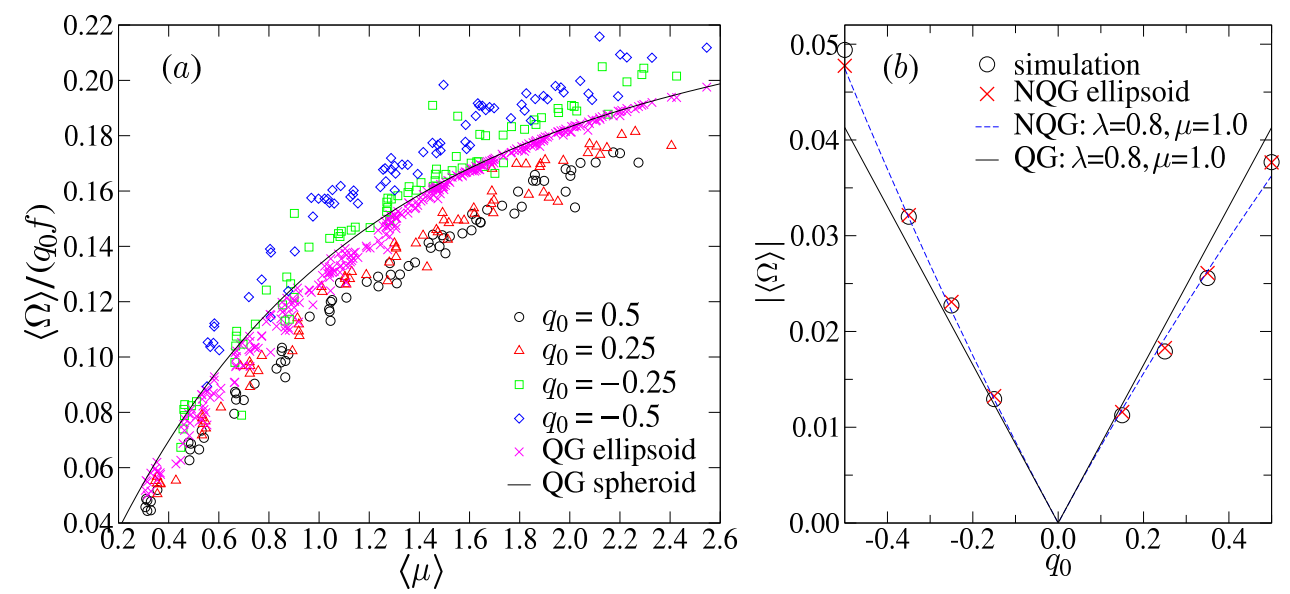

FIGURE 6. (a) Scatter plot showing the correlation between the rotation rate $\langle\Omega\rangle$ (normalised by $q_{0} f$ ) and the vertical aspect ratio $\langle\mu\rangle$ when the vortex is in approximate equilibrium at large time. For each simulation, we also calculate the corresponding QG ellipsoid rotation rate $\Omega_{\mathrm{QG}}(\langle\lambda\rangle,\langle\mu\rangle)$ using (4.1) (its normalised values are plotted as crosses). For reference, the rotation rate of a QG spheroid $\Omega_{\mathrm{QG}}(\lambda=1, \mu)$ is included as a solid line. $(b)|\langle\Omega\rangle|$ versus $q_{0}$ for selected simulations having similar aspect ratios (circles). At each $q_{0}$, the vortex has $\{\langle\lambda\rangle,\langle\mu\rangle\}$ within $10 \%$ of $\{0.8,1.0\}$. The rotation rate of an NQG ellipsoid (see text) with the same aspect ratios $\{\langle\lambda\rangle,\langle\mu\rangle\}$ is plotted by a cross for each $q_{0}$. The dashed and solid lines give, respectively, the rotation rate of an NQG ellipsoid and a QG ellipsoid, both having aspect ratios $\{\lambda, \mu\}=\{0.8,1.0\}$. Only the QG rotation rate is symmetric about $q_{0}=0$.

cases in the principal data set. The corresponding QG ellipsoid rotation rate for each case, i.e., $\Omega_{\mathrm{QG}}(\langle\lambda\rangle,\langle\mu\rangle)$, is also included in the plot.

A remarkable feature of figure 6(a) is that the data for each $q_{0}$ strongly clusters around a single curve, implying that $\langle\lambda\rangle$ plays only a minimal role here. On the other hand, $\langle\Omega\rangle$ shows a strong 


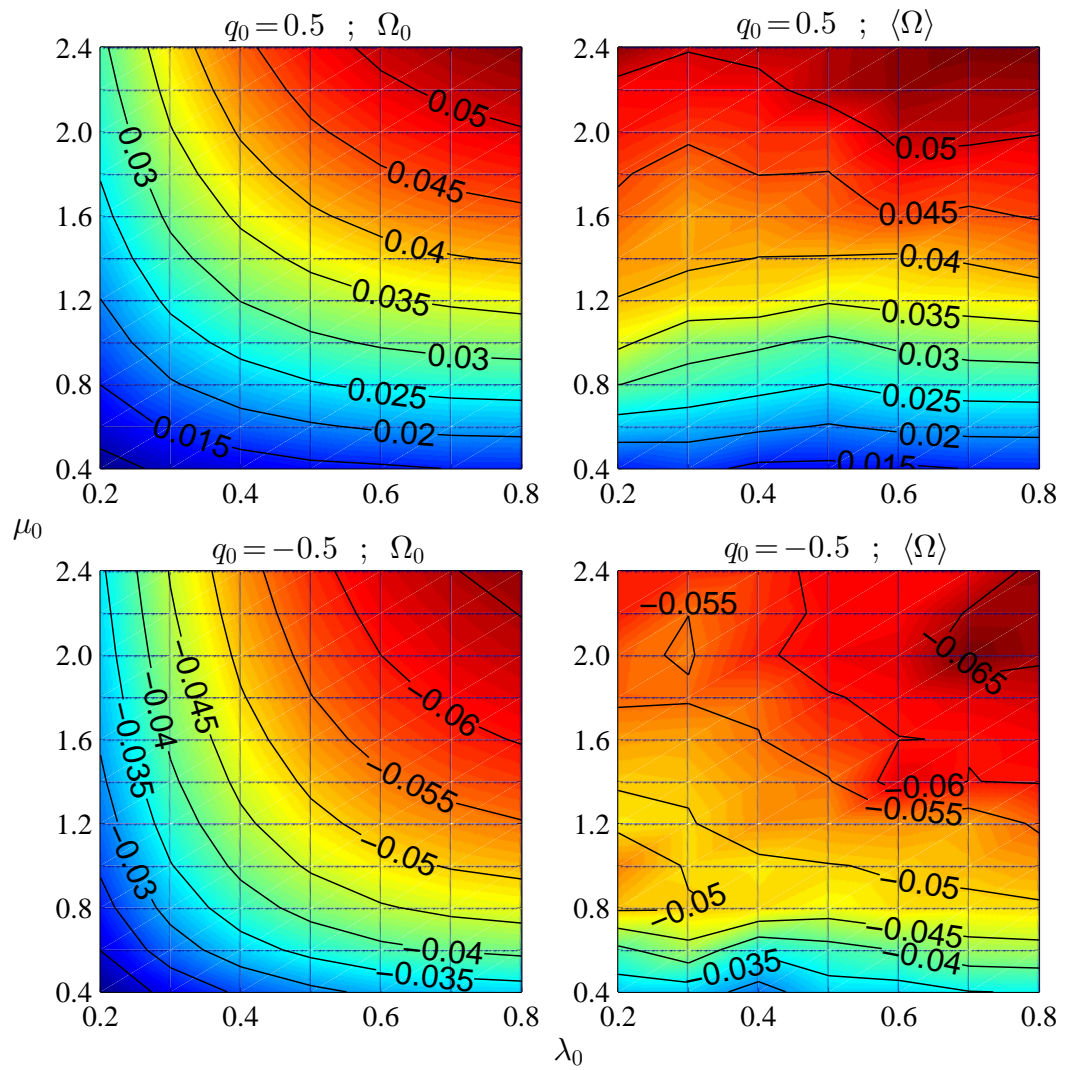

FIGURE 7. The initial rotation rate $\Omega_{0}$ at time $t=0$ (left) and the average rotation rate $\langle\Omega\rangle$ of the equilibrium state at large $t$ (right) as a function of the initial aspect ratios $\lambda_{0}$ and $\mu_{0}$ for $q_{0}= \pm 0.5$ (top and bottom). For a quasi-stable vortex, $\langle\Omega\rangle \approx \Omega_{0}$. Assuming $\langle\Omega\rangle$ depends mainly on $\langle\lambda\rangle$ and $\langle\mu\rangle$ but not on the exact vortex shape (at least when $\langle\varrho\rangle \approx 1$ ), then $\langle\Omega\rangle$ for an unstable vortex should be close to the rotation rate of a quasi-stable ellipsoid with the same $\langle\lambda\rangle$ and $\langle\mu\rangle$. Hence, pictorially speaking, the right panels are obtained by flattening out the contours in the left panels. For $q_{0}=0.5$, the weak dependence of $\langle\Omega\rangle$ on $\lambda_{0}$ reflects the fact that $\langle\mu\rangle \approx \mu_{0}$ as evident in figure 4 . For $q_{0}=-0.5$, the small irregularities in $\langle\Omega\rangle$ around $(0.2,1.0)$ and $(0.3,2.0)$ are caused by the significant variation in the vertical aspect ratio $\mu$ in these regions, as seen from figure 4 . Cases with $q_{0}= \pm 0.25$ show similar behaviour.

dependence on $\langle\mu\rangle$. This is a consequence of the evolutionary changes exhibited by $\langle\lambda\rangle$ and $\langle\mu\rangle$ in figure 4. To elucidate, we plot $\Omega_{0} \equiv \Omega(t=0)$ and $\langle\Omega\rangle$ as a function of the initial aspect ratios $\lambda_{0}$ and $\mu_{0}$ in figure 7 (see Appendix B for the method to compute $\Omega_{0}$ ). For a quasi-stable vortex, $\langle\Omega\rangle \approx \Omega_{0},\langle\lambda\rangle \approx \lambda_{0}$ and $\langle\mu\rangle \approx \mu_{0}$ since the initial ellipsoidal shape is maintained. For an unstable vortex, figure 4 and figure 7 together suggest that at late times, $\langle\Omega\rangle$ is close to the rotation rate of a quasi-stable ellipsoid with the same $\langle\lambda\rangle$ and $\langle\mu\rangle$. In other words, for a vortex whose aspect ratios evolve from $\left\{\lambda_{0}, \mu_{0}\right\}$ to $\{\langle\lambda\rangle,\langle\mu\rangle\}$, the late time average rotation rate $\langle\Omega\rangle$ is well estimated by $\Omega_{0}\left(\lambda_{0}=\langle\lambda\rangle, \mu_{0}=\langle\mu\rangle\right)$. This can be seen by comparing the left and right panels of figure 7 (see also figure 5(a) for an example). The fact that $\{\langle\lambda\rangle,\langle\mu\rangle\}$ mostly clusters around the stability boundary together with the observation that $\Omega_{0}$ depends weakly on $\lambda_{0}$ for $0.5 \leqslant \lambda_{0} \leqslant 1$ imply that $\langle\lambda\rangle$ is not important in determining $\langle\Omega\rangle$. It also follows that the $\langle\mu\rangle-$ dependence of $\langle\Omega\rangle$ seen in figure 6(a) is well approximated by $\Omega_{0}\left(\lambda_{0}, \mu_{0}=\langle\mu\rangle\right)$ for some fixed $\lambda_{0} \approx 1$. 
Figure 6(a) also reveals that ageostrophic effects occurring at finite Rossby number result in a visible asymmetry between cyclones and anticyclones: for the same aspect ratios $\langle\lambda\rangle$ and $\langle\mu\rangle$ and PV anomaly $\left|q_{0}\right|$, an anticyclone rotates faster than a cyclone. The corresponding $\left|\Omega_{\mathrm{QG}}\right|$ denoted by crosses in figure 6(a) lies somewhere between the cyclonic and the anticyclonic rotation rates. Cyclone-anticyclone asymmetry can be traced back to the nonlinear terms in (2.16) which break the invariance of the PV inversion under the transformation $(q, \varphi) \rightarrow-(q, \varphi)$. Moreover this asymmetry can be quantitatively captured by a next-order correction to QG theory, as shown next.

\subsection{Vortex rotation rate estimated by a higher-order balanced model}

A number of balanced models have been developed to extend the QG approximation (see e.g. Warn et al. (1995), Muraki et al. (1999) and references therein). Here, we adopt the nonlinear quasi-geostrophic (NQG) balanced model of McKiver \& Dritschel (2008), see Appendix C. The NQG balanced model uses a Rossby number expansion to second-order, $\mathcal{O}\left(q_{0}^{2}\right)$, except it retains the exact definition of $P V$. This greatly improves the estimate of the underlying balanced flow, as demonstrated explicitly in McKiver \& Dritschel (2008). The resulting nonlinear PV inversion relations allow one to reconstruct the velocity and buoyancy fields to order $\mathcal{O}\left(q_{0}^{2}\right)$ from a given instantaneous PV field.

In figure $6(b)$, we plot $|\langle\Omega\rangle|$ versus $q_{0}$ for several cases having similar aspect ratios $\langle\lambda\rangle$ and $\langle\mu\rangle$. Corresponding to each of these cases, we construct the velocity field from an ellipsoid of uniform PV with the same $\langle\lambda\rangle,\langle\mu\rangle$ and $q_{0}$ by NQG PV inversion. The rotation rates of such NQGbalanced ellipsoids are then calculated (see Appendix B). We find close agreement, typically within $3 \%$, between the theoretical NQG rotation rate and the data across all $q_{0}$. Figure $6(b)$ also shows the rotation rate of an NQG ellipsoid with $\lambda=0.8$ and $\mu=1.0$ as a function of $q_{0}$. These results clearly reveal the emergence of asymmetry in the cyclonic and anticyclonic rotation rate as soon as finite Rossby number effects are taken into account.

\subsection{Discussion}

We have seen above that $\Omega$ has a strong dependence on the vertical aspect ratio $\mu$. Moreover, ageostrophic effects at $\mathcal{O}\left(q_{0}^{2}\right)$ make anticyclones spin faster than cyclones, particularly at large $\mu$ (see figure 6). In observations, for the cyclonic Gulf Stream cold core ring reported in Spence \& Legeckis $(1981)\left(\lambda=0.65, \mu=1.10, q_{0}=0.52\right.$ and $\left.f=8.6 \times 10^{-5}\right)$, we estimate $\Omega / \Omega_{\mathrm{QG}}=$ $0.73<1$; while for the anticyclonic meddy documented by Hebert et al. (1990) $(\lambda=0.71$, $\mu=0.32, q_{0}=0.35$ and $\left.f=1.0 \times 10^{-4}\right)$, we estimate $\Omega / \Omega_{\mathrm{QG}}=1.03 \gtrsim 1$. These two individual cases appear to support our findings. Exceptionally, the anticyclonic meddy "Ulla" has $\Omega / \Omega_{\mathrm{QG}}<$ 1. This is perhaps not surprising as Ulla "is not just another meddy" (Paillet et al. 2002) and has some peculiar features. In order to fully verify the $\mathcal{O}\left(q_{0}^{2}\right)$ effects discussed here, laboratory experiments under controlled conditions are probably needed. Additionally, there appears to be a wealth of untapped numerical simulation data available from high-resolution ocean models. This should be exploited.

\section{Balance}

\subsection{Definitions and method of analysis}

The control exerted by PV on the dynamics of vortices in rotating, stably-stratified geophysical flows can be, for all practical purpose, nearly complete, with inertia-gravity waves (IGWs) playing a negligible role. This state of affairs is known as 'balance'. Balance is favoured when the magnitude of the Rossby number $\left|q_{0}\right| \lesssim 1$ and the frequency ratio $f / N \lesssim 1$, even in strongly 
turbulent flows (Dritschel \& Viúdez 2007; Dritschel \& McKiver 2014) — at least for carefully prepared initial conditions.

As described in $\S 2.4$, we initialise each flow by artificially ramping up the PV anomaly over many inertial periods while otherwise solving the full equations of motion. This results in an 'initial' state nearly void of IGWs when $\left|q_{0}\right| \lesssim 1$ and $f / N \lesssim 1$. To explore the possible spontaneous emission of IGWs at later times and to estimate their contribution to the dynamics, we need to quantify balance and the residual imbalance or IGW activity. One approach to diagnose the balanced component from a given PV field is to invert the 'balance conditions' obtained from a Rossby number expansion of the governing equations. Such balance conditions define an instantaneous state of balance. The traditional QG balance and the higher-order NQG balance in $\S 4.1$ fall into this category. Here, we take another approach, called Optimal Potential Vorticity (OPV) balance (Viúdez \& Dritschel 2004), which does not enforce any specific balanced conditions but instead seeks the 'minimally imbalanced' state through a numerical iterative procedure.

Given the PV distribution at some chosen diagnostic time $t$, the OPV procedure carries out a series of artificial forward and backward integrations of the equations of motion, over a specified time period $\tau$. Each integration involves ramping up or ramping down the PV anomaly. The objective is to arrive at the original given PV distribution from a different one in a state of rest at time $t-\tau$, after ramping up the PV anomaly and otherwise solving the full equations of motion. The resulting fields are called 'balanced', even though they may contain small residual imbalance (Dritschel \& Viúdez 2007; Viúdez 2007). Note, the balance depends only on the PV distribution provided and the ramp period $\tau$. The choice of $\tau$ is strongly affected by the amount of distortion of the PV distribution occurring between $t-\tau$ (the base distribution) and $t$ (the given target distribution) during the ramp. If $\tau$ is too long, the base distribution is too distorted to ever converge to the target one. A compromise must be made. Following previous studies (Dritschel \& Viúdez 2007; McKiver \& Dritschel 2008) and after conducting further tests here, we took $\tau$ to be 4 inertial periods. This is sufficiently long not to excite significant IGW activity, though not long enough to avoid all of it. In this sense, it is better to view OPV balance as a procedure which seeks the minimally imbalanced state, subject to the constraint that the base PV distribution can actually evolve into the target PV distribution. The technical details of OPV balance are summarised in Appendix D.

Given the OPV balanced flow $\varphi_{\text {bal }}$ at a chosen diagnostic time $t$, the imbalanced flow (or 'imbalance') is defined simply by subtraction from the full flow,

$$
\varphi_{\text {imb }}=\varphi-\varphi_{\text {bal }} .
$$

The balanced flow is further divided into a $Q G$ balanced part $\varphi_{Q G}=\left(0,0, \phi_{Q G}\right)$ and the remaining 'ageostrophic' balanced part

$$
\varphi_{\mathrm{AGb}}=\varphi_{\mathrm{bal}}-\varphi_{\mathrm{QG}} .
$$

Here, $\phi_{\mathrm{QG}}$ is defined in the standard way, inverting the QG linear operator on the PV anomaly $q$ (see (C 3) in Appendix C) on flat isopycnal surfaces, not on the actual displaced surfaces (the displacement is proportional to $q_{0}$, and the effect of this displacement is $\mathcal{O}\left(q_{0}^{2}\right)$ on the balanced flow - therefore an ageostrophic effect).

We next focus on two cases which serve to illustrate how the nature of both the balanced flow and the imbalance vary with the vortex shape and the Rossby number $q_{0}$. In each case, we examine a time at or near the end of the simulation when the vortex is in a near equilibrium state. As discussed below, the imbalance is often largest during a period of instability and rapid change, though it never contributes significantly to the total energy. We finish by examining the fractional energy associated with the balanced and imbalanced flow components as a function of the Rossby number $q_{0}$. 


\subsection{Oblate, weakly-eccentric vortices}

We first look at an oblate cyclone and anticyclone at $\left|q_{0}\right|=0.25$ with $\lambda_{0}=0.8$ and $\mu_{0}=0.4$. These vortices are quasi-stable: in the late-time equilibrium state $\langle\lambda\rangle \approx \lambda(t=0)=0.81$ in both cases, and $\langle\mu\rangle \approx \mu(t=0)=0.35$ and 0.46 for the cyclone and anticyclone respectively.

The top row of figure 8 shows the balanced and imbalanced components of the scaled vertical vorticity $\zeta / f$ for the cyclone while the bottom row shows the analogous fields for the anticyclone. First of all, the QG balanced fields are entirely as expected: positive vorticity inside the vortex for the cyclone and negative for the anticyclone. Just outside, the vorticity switches sign. Again, this is consistent since, inside the cyclone, vortex lines are more bunched together, while outside they must be more spread out in order to relax to the undisturbed state with absolute vorticity $f \hat{\boldsymbol{k}}$ far from the vortex. The anticyclone is exactly the opposite. On the other hand, the ageostrophic balanced fields do not preserve the anti-symmetry between cyclones and anticyclones. In both cases, $\zeta_{\text {AGb }}$ is negative inside the vortex, and weakly positive just outside of it. This is because $\zeta_{\text {AGb }}$ depends quadratically, to leading order, on the QG balanced fields (see Appendix C or, explicitly, (2.23) of McKiver \& Dritschel (2008)).

Turning to the imbalanced vertical vorticity $\zeta_{\text {imb }}$ (right panels of the figure), we see much smaller-scale features, strongest at the edge or inside the vortex. These features resemble wave crests running principally diagonally, as one would expect of IGWs 'forced' at sub-inertial frequencies (note the St Andrews cross at the centre of the cyclone). The forcing is provided by the rotating, distorted vortex, and we see here evidence of a continuous spontaneous emission of IGWs by the balanced vortical flow. Some wave trapping is evident in the anticyclone, but the amplitude of the IGWs is closely comparable for both vortices. The amplitudes of the waves are exceedingly weak; imbalanced field values are $10^{2}$ to $10^{3}$ times smaller than balanced field values at this Rossby number, $\left|q_{0}\right|=0.25$. This remains true even at $\left|q_{0}\right|=0.5$ (see $\S 5.4$ for a discussion of the Rossby number scaling).

Analogous results for the anomalous 'static stability',

$$
\gamma=\frac{1}{N^{2}} \frac{\partial b}{\partial z}
$$

are presented in figure 9 (top row for the cyclone and bottom row for the anticyclone). Note that $\gamma$ is the negative of the vertical derivative of the isopycnal displacement. The local squared buoyancy frequency equals $N^{2}(1+\gamma)$, so $\gamma<-1$ indicates static instability (overturning isopycnals). The structure of $\gamma_{Q G}$ in the vortex interior implies increased stability $(\gamma>0)$ for cyclones, and decreased stability $(\gamma<0)$ for anticyclones. Note that $\gamma_{\mathrm{QG}}$ has the same sign as $\zeta_{\mathrm{QG}} / f$ in the vortex interior, but the opposite sign outside. The balanced, ageostrophic component $\gamma_{\mathrm{AGb}}$ (at second order in Rossby number) has almost no effect on stability within the vortex. Only at the vortex edge is $\gamma$ reduced, but it is increased above and below it, for both the cyclone and the anticyclone.

The imbalanced field $\gamma_{\text {imb }}$ again displays evidence of IGW activity, more pronounced above and below the anticyclone (which is less oblate than the cyclone at this time). The pattern of $-\gamma_{\text {imb }}$ is remarkably similar to $\zeta_{\mathrm{imb}} / f$. In fact, for plane-wave solutions of the linearised equations about a state of rest, these two fields exactly cancel. The sum $\zeta_{\mathrm{imb}} / f+\gamma_{\mathrm{imb}}$ is indeed much smaller outside the vortex than either field in isolation, as seen in figure 10 displaying onedimensional cross sections from the two fields of the cyclone. This confirms that the imbalanced fields mainly consist of IGWs.

The vertical velocity $w$ contains only a balanced ageostrophic component $w_{\text {AGb }}$ and the imbalanced one $w_{\text {imb }}$. These are shown in figure 11 (for the cyclone) and figure 12 (for the anticyclone). The balanced component $w_{\mathrm{AGb}}$ displays a three-dimensional octopolar structure, typical of a rotating ellipsoid around which isopycnals rise and fall anti-symmetrically about the midplane 


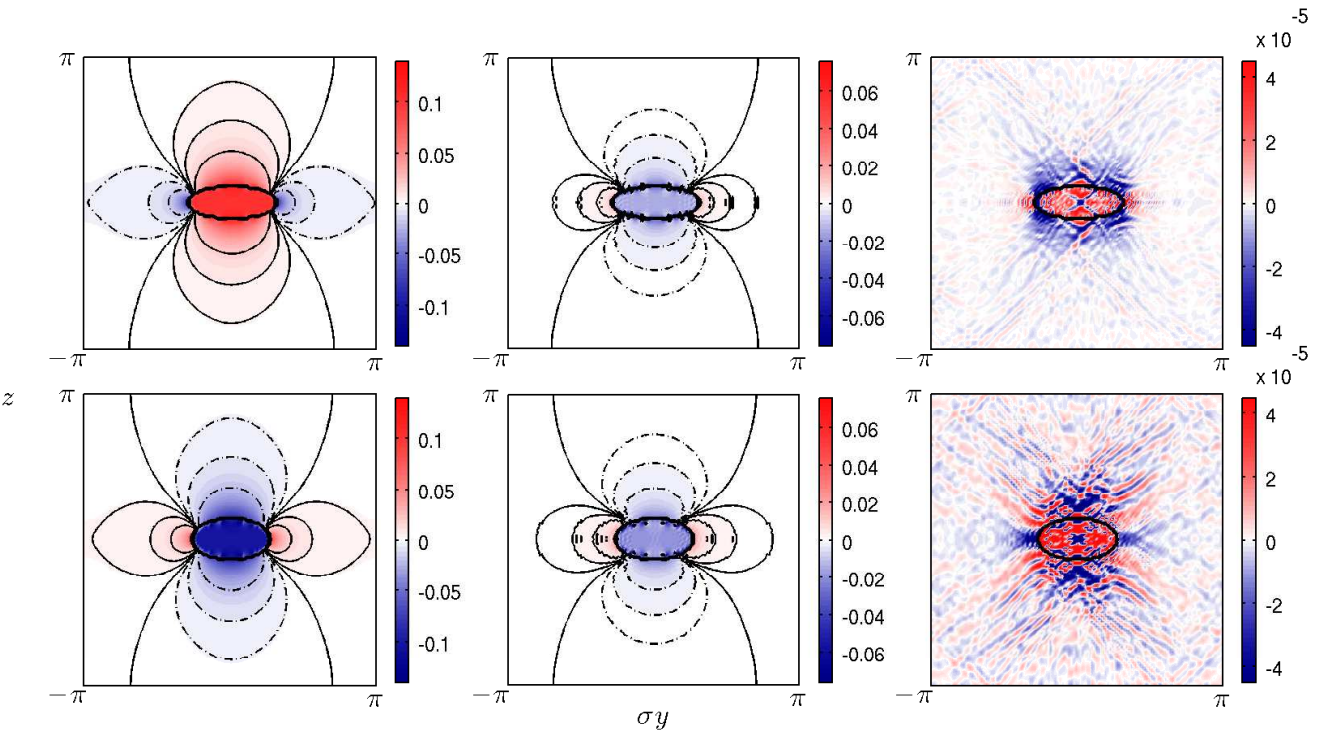

FIGURE 8. A vertical $y$ - $z$ cross section at $x=-\sigma^{-1} \pi / 32$ of the scaled vertical vorticity $\zeta / f$ for a cyclonic vortex or 'cyclone' (top row) and an anticyclonic vortex or 'anticyclone' (bottom row) with $q_{0}= \pm 0.25$ respectively. The initial aspect ratios are $\lambda_{0}=0.8$ and $\mu_{0}=0.4$ in both cases. The QG balanced part $\zeta_{\mathrm{QG}} / f$ is on the left, the ageostrophic balanced part $\zeta_{\mathrm{AGb}} / f$ is in the middle and the imbalanced part $\zeta_{\mathrm{imb}} / f$ is on the right. The fields are depicted at $t=480 T_{\mathrm{ip}}$. The bold black contour indicates the vortex edge, while the thin solid and thin dashed contours in the left two panels denote positive and negative field values, respectively. Note: the plotted domain is squeezed horizontally by the factor $\sigma=f / N=0.1$. A nonlinear colour scale is used for the imbalanced fields to emphasise the weaker wave structure outside the vortex. The actual maximum of $\left|\zeta_{\text {imb }} / f\right|$ is about $8 \times 10^{-4}$.

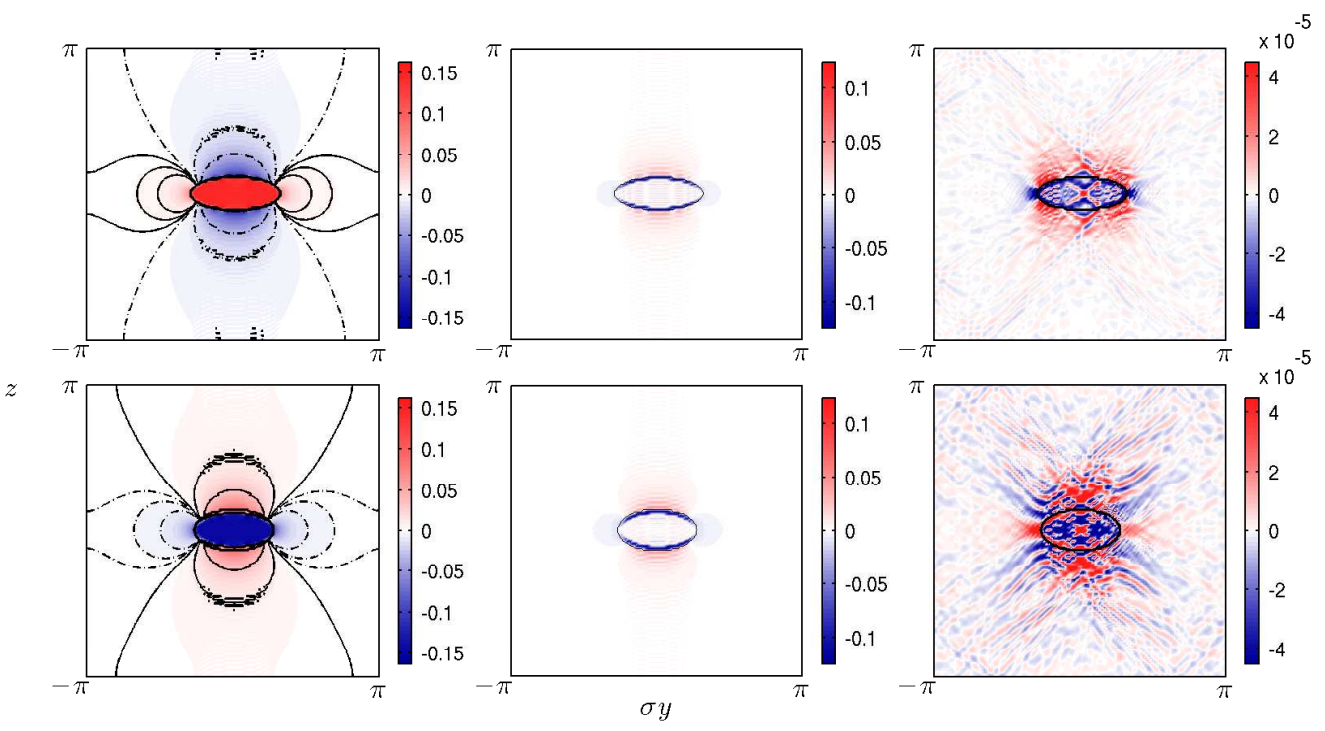

FIGURE 9. As in figure 8 except for the anomalous 'static stability' $\gamma=b_{z} / N^{2}$, with the QG balanced part $\gamma_{\mathrm{QG}}$ on the left, the ageostrophic balanced part $\gamma_{\mathrm{AGb}}$ in the middle, and the imbalanced part $\gamma_{\text {imb }}$ on the right. The top row is for the cyclone and the bottom row is for the anticyclone. A nonlinear color scale is used for the imbalanced fields. The maximum $\left|\gamma_{\mathrm{imb}}\right|$ is about $6 \times 10^{-4}$. 

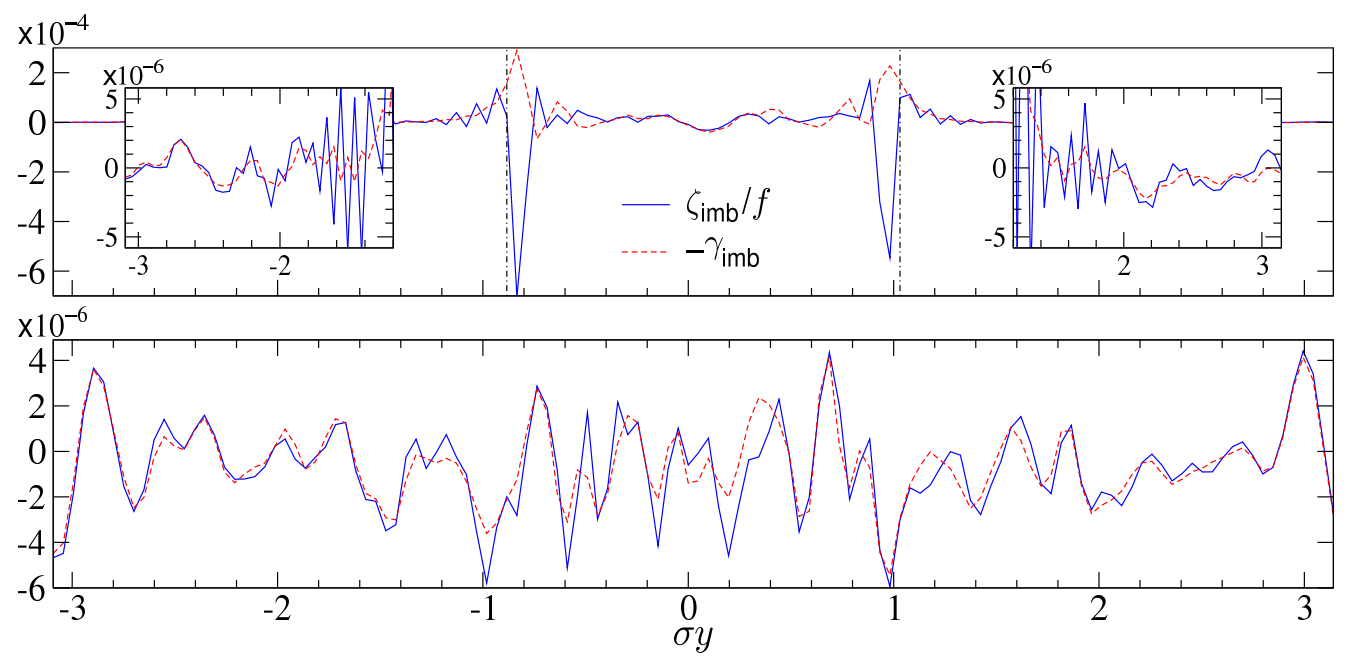

FIGURE 10. One-dimensional cross sections along $y$ of $\zeta_{\mathrm{imb}} / f$ (solid) and $-\gamma_{\mathrm{imb}}$ (dashed) for the oblate cyclone shown in figure 8 . The vertical dot-dashed lines indicate the location of the vortex edge. Top: a transect through the vortex at $(x, z)=\left(-\sigma^{-1} \pi / 32,0\right)$ (inserts show regions having much weaker fields). Bottom: a transect below the vortex at $(x, z)=\left(-\sigma^{-1} \pi / 32,-\pi / 2\right)$. The relation $\zeta_{\text {imb }} / f+\gamma_{\text {imb }}=0$ satisfied by plane-wave IGWs holds well, verifying the imbalanced fields mainly consist of IGWs.

$z=0$ as the vortex rotates. In $w$, the cyclone exhibits a significantly higher degree of balance, with the magnitude of $w_{\text {imb }}$ being nearly 10 times smaller than $w_{\mathrm{AGb}}$. For the anticyclone, by contrast, $w_{\mathrm{AGb}}$ and $w_{\text {imb }}$ are comparable (note that the color scales for the imbalanced fields in the figures are nonlinear), and it is evident that OPV balance struggles to clearly define balance. In both flows, waves appear to be trapped inside the vortex, especially in the horizontal cross sections. Wave crests run approximately perpendicular to the flow at the vortex edge, but become radial approaching the centre of the vortex. While the imbalance is particularly visible here, it nonetheless plays an insignificant role in the flow evolution. This echoes previous findings when care is taken to carefully balance the initial conditions (Dritschel \& Viúdez 2007; McKiver \& Dritschel 2008).

\subsection{Prolate vortices}

We next compare the fields associated with two highly-prolate vortices $\left(\lambda_{0}=0.7, \mu_{0}=2.4\right.$ and $\left.q_{0}= \pm 0.25\right)$ with the oblate vortices examined just above. These prolate vortices are unstable and undergo mild filament shedding at the top and bottom. At equilibrium, $\{\langle\lambda\rangle,\langle\mu\rangle\}=$ $\{0.95,2.21\}$ and $\{0.91,2.29\}$ for the cyclone and anticyclone respectively.

Figure 13 shows components of vertical vorticity, $\zeta_{\mathrm{QG}}, \zeta_{\mathrm{AGb}}$ and $\zeta_{\mathrm{imb}}$ (normalised by $f$ ), for a cyclone and an anticyclone (top and bottom rows). Comparing with the oblate vortices in figure 8 , we observe broadly similar features, especially in the balanced components. The ageostrophic component again decreases $\zeta$ in the vortex core in both cases, but the decrease is less here due to the more barotropic character of the vortices. The imbalance $\zeta_{\mathrm{imb}}$ is also more confined to the vortex interior. In all cases, one sees a characteristic St Andrews cross pattern associated with IGWs emanating from the rotating, non-axisymmetric vortex. Note however that these waves are exceptionally weak, carrying vorticity nearly $10^{3}$ times smaller than the balanced component.

Regarding the anomalous static stability $\gamma=b_{z} / N^{2}$ in figure 14 , the balanced components are broadly similar to those shown for the oblate vortices in figure 9: QG balance enhances the stability of cyclones and reduces the stability of anticyclones. The ageostrophic component $\gamma_{\text {AGb }}$ 

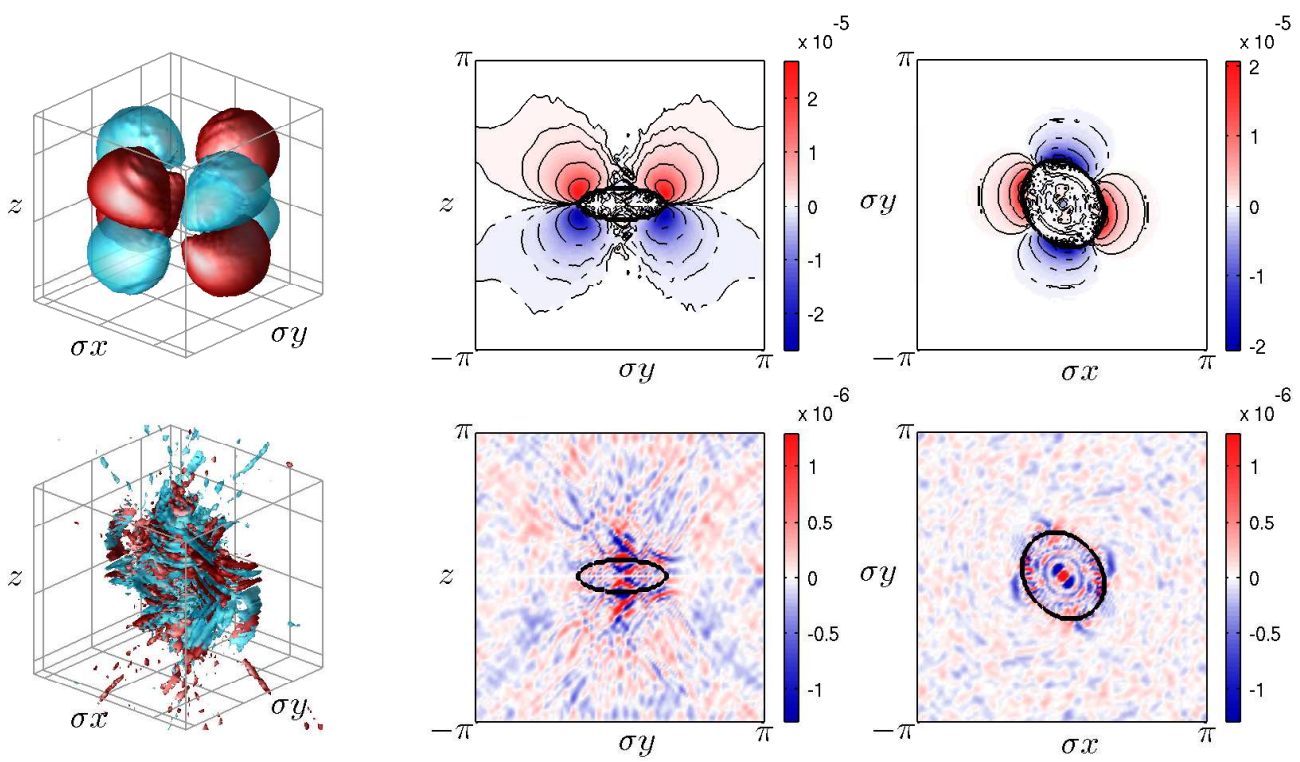

FIGURE 11. The ageostrophic balanced part $w_{\mathrm{AGb}}$ (top row) and the imbalanced part $w_{\text {imb }}$ (bottom row) of the vertical velocity associated with a cyclonic oblate vortex $\left(q_{0}=0.25, \lambda_{0}=0.8\right.$ and $\left.\mu_{0}=0.4\right)$. The left column shows three-dimensional surfaces at $w_{\mathrm{AGb}}= \pm 5 \times 10^{-6}$ and $w_{\text {imb }}= \pm 5 \times 10^{-7}$ (positive values are in red). The middle column shows a vertical $y$ - $z$ cross section at $x=-\sigma^{-1} \pi / 32$. The right column is a horizontal $x-y$ cross section at $z=-\pi / 32$. A nonlinear color scale is used in the $w_{\text {imb }}$ cross section plots. The maximum $\left|w_{\text {imb }}\right|$ is about $5 \times 10^{-6}$.
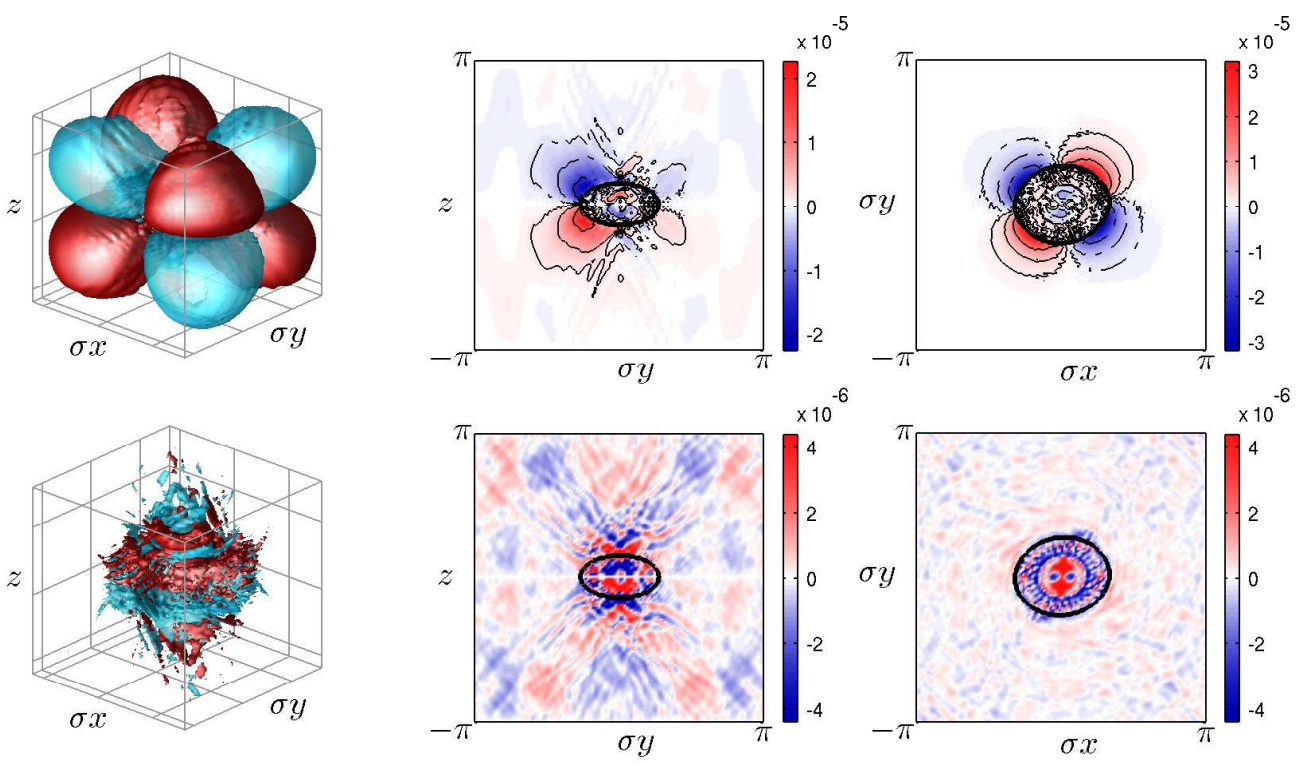

FIGURE 12. As in figure 11 but for an anticyclonic vortex with $q_{0}=-0.25$. Surfaces at $w_{\text {AGb }}= \pm 5 \times 10^{-6}$ and $w_{\text {imb }}= \pm 2 \times 10^{-6}$ are shown in the left column. A nonlinear color scale is used in the $w_{\text {imb }}$ cross section plots. The maximum $\left|w_{\text {imb }}\right|$ is about $2 \times 10^{-5}$. 


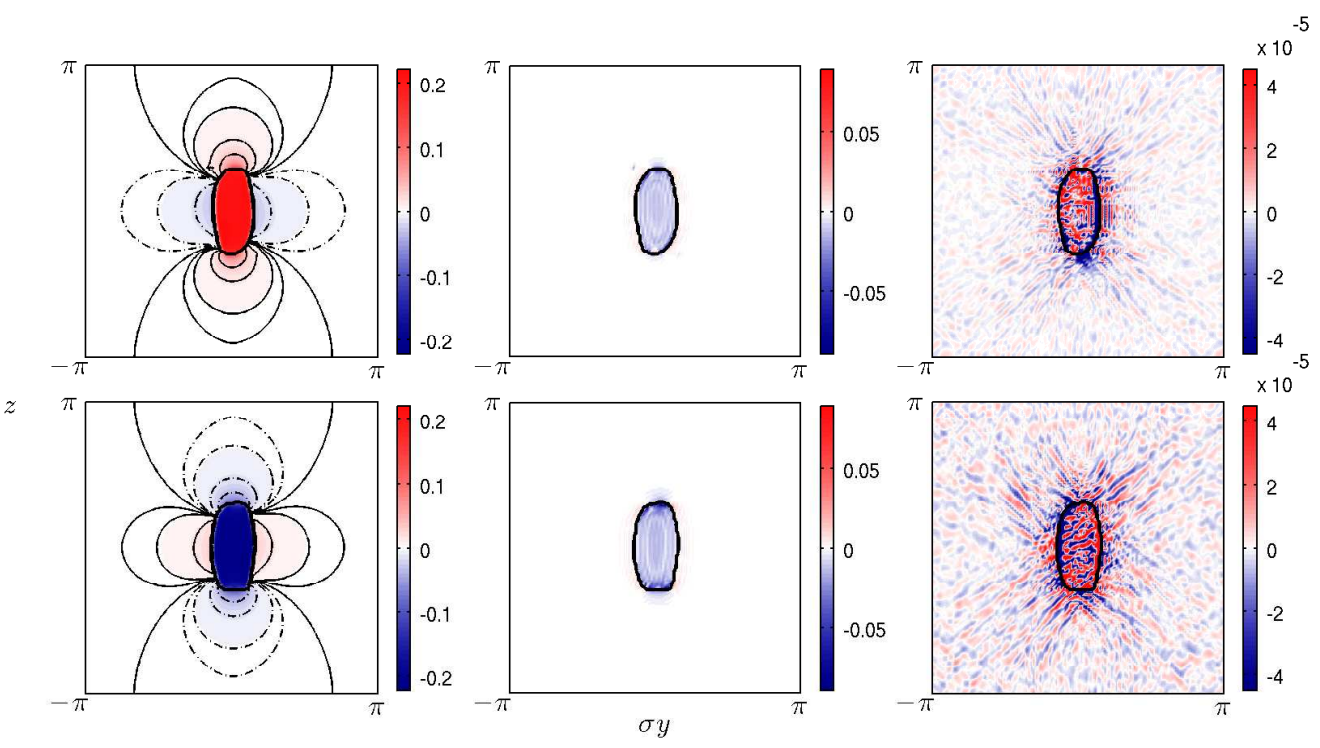

FIGURE 13. A vertical $y$ - $z$ cross section at $x=-\sigma^{-1} \pi / 32$ of the scaled vertical vorticity $\zeta / f$ for a cyclone (top row) and an anticyclone (bottom row) with $q_{0}= \pm 0.25$ respectively. The initial aspect ratios are $\lambda_{0}=0.7$ and $\mu_{0}=2.4$ in both cases. The layout is the same as in figure 8 with the QG balanced part $\zeta_{\mathrm{QG}} / f$ on the left, the ageostrophic balanced part $\zeta_{\mathrm{AGb}} / f$ in the middle, and the imbalanced part $\zeta_{\mathrm{imb}} / f$ on the right. The fields are depicted at $t=1200 T_{\mathrm{ip}}$. A nonlinear color scale emphasising the weak wave pattern outside the vortex is used for the imbalanced fields. The actual maximum of $\left|\zeta_{\text {imb }} / f\right|$ is about $8 \times 10^{-4}$.

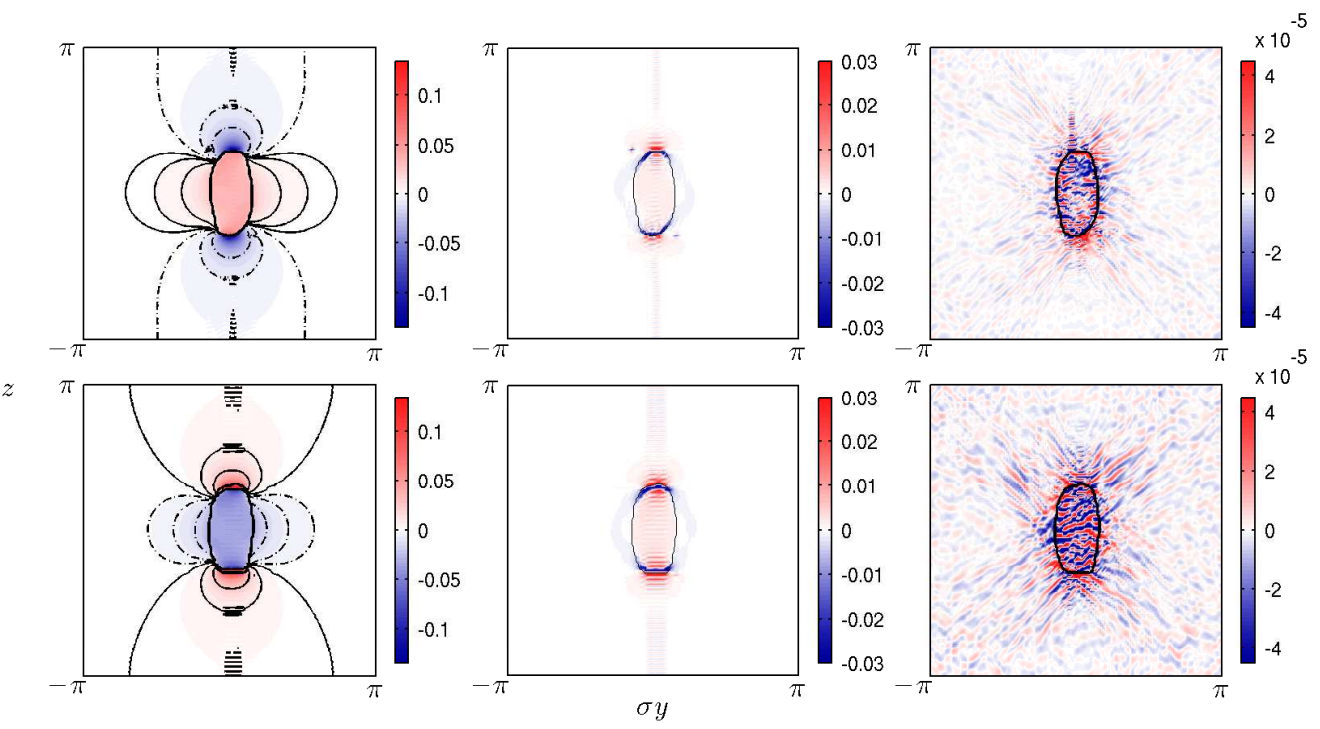

FIGURE 14. As in figure 13 except for the anomalous static stability $\gamma=b_{z} / N^{2}$, with the QG balanced part $\gamma_{\mathrm{QG}}$ on the left, the ageostrophic balanced part $\gamma_{\mathrm{AGb}}$ in the middle, and the imbalanced part $\gamma_{\text {imb }}$ on the right. The top row is for the cyclone and the bottom row is for the anticyclone. A nonlinear color scale is used for the imbalanced fields. The maximum $\left|\gamma_{\text {imb }}\right|$ is about $2 \times 10^{-3}$. 
is insignificant within the vortex and has a minor effect on stability. Regarding the imbalance, $\gamma_{\text {imb }}$ has a similar structure to $\zeta_{\text {imb }} / f$, as expected for pure IGWs, and again the imbalance contributes negligibly to the dynamics ( $\gamma_{\text {imb }}$ is $10^{2}$ times smaller than $\gamma_{Q G}$ ).

The vertical velocity components $w_{\mathrm{AGb}}$ and $w_{\mathrm{imb}}$, shown in figure 15 (for the cyclone) and in figure 16 (for the anticyclone), are overall significantly different from their oblate-vortex counterparts in figures 11 and 12. First, the ageostrophic component is less well organised and more concentrated in the interior of prolate vortices, perhaps due to fact that vertical motions are less constrained. The pattern is much more complex, especially for the cyclonic state, where no octopolar pattern is evident. The imbalance $w_{\text {imb }}$ displays the characteristic St Andrews cross pattern in the $y-z$ cross section, but in the $x-y$ cross section wave crests are seen to be predominantly radial switching abruptly to azimuthal just outside the vortex boundary. This structure is not seen clearly for the oblate vortices examined, and is likely due to the greater horizontal distortion of the vortex. Finally, as a fraction of $w_{\mathrm{AGb}}$, the imbalance $w_{\mathrm{imb}}$ is appreciably weaker for these prolate vortices compared to their oblate counterparts. Here, $w_{\text {imb }}$ is 20 to 30 times smaller than $w_{\text {AGb }}$. Even in vertical velocity, these flows exhibit a strong degree of balance.

\subsection{Energetics and Rossby number scaling}

We next quantify the importance of the balanced and imbalanced parts of the flow using the energy norm

$$
E_{i}=\frac{1}{2}\left\langle\left|\boldsymbol{u}_{i}\right|^{2}+b_{i}^{2} / N^{2}\right\rangle .
$$

where $i=\mathrm{QG}, \mathrm{AGb}$ or $\mathrm{imb}$, and in this section $\langle\cdot\rangle$ denotes a domain average. We consider two cases, each having the same initial aspect ratios $\lambda_{0}=0.2$ and $\mu_{0}=2.4$, but opposite PV, $q_{0}= \pm 0.5$. Also, the simulations are carried out at double the standard resolution in each direction (i.e. at $256^{3}$ with subgrid PV kept to scales 16 times finer), and using a time step $\Delta t=0.075$. These prolate and initially highly-eccentric vortices quickly become unstable and exhibit strong filament shedding between $t \approx 200 T_{\mathrm{ip}}$ and $t \approx 1500 T_{\mathrm{ip}}$ before finally settling down with $\{\langle\lambda\rangle,\langle\mu\rangle\}=\{0.90,2.29\}$ and $\{0.88,1.73\}$ for the cyclone and anticyclone respectively.

Figure 17 shows the time evolution of the energy ratios $E_{\mathrm{QG}} / E_{\mathrm{tot}}, E_{\mathrm{AGb}} / E_{\mathrm{tot}}$ and $E_{\mathrm{imb}} / E_{\mathrm{tot}}$ where $E_{\text {tot }}$ is the total energy, found from (5.4) using the full fields $\boldsymbol{u}$ and $b$. Notably, $E_{\mathrm{QG}}>E_{\text {tot }}$ and generally increases in time for the cyclone; just the opposite is true for the anticyclone. Hence, QG balance overestimates the energy of cyclones and underestimates that of anticyclones (this has been found to be true generally in all cases examined). We also find that oblate cyclones have larger $E_{\mathrm{QG}} / E_{\text {tot }}(>1)$ than prolate ones, while oblate anticyclones have smaller $E_{\mathrm{QG}} / E_{\text {tot }}$ $(<1)$ than prolate ones. In other words, increasing vortex oblateness increases the difference $\left|E_{\mathrm{QG}} / E_{\text {tot }}-1\right|$.

Turning to the ageostrophic balanced part $E_{\mathrm{AGb}}$, this is seen to be just $1 \%$ to $2 \%$ of $E_{\text {tot }}$ even at these moderately high Rossby numbers. $E_{\mathrm{AGb}} / E_{\text {tot }}$ generally grows in time and appears to level off at late times. Note that two estimates of $E_{\mathrm{AGb}}$ are provided in the figure (middle column), one obtained using OPV balance and the other using NQG balance. Both give closely comparable measures of the small ageostrophic balanced part. Note, simple scaling estimates suggest $E_{\mathrm{AGb}} / E_{\text {tot }} \sim q_{0}^{2}$ (see below), which overestimates the observed energy ratio by more than an order of magnitude if one naively assumes an $\mathcal{O}(1)$ prefactor in the scaling.

Finally, regarding the imbalanced part $E_{\mathrm{imb}}$, first of all it is much smaller than even $E_{\mathrm{AGb}}$, with energy ratios of the order $10^{-5}$ or smaller. $E_{\mathrm{imb}} / E_{\mathrm{tot}}$ is largest when the vortex undergoes deformation at early times and decays to very small values at late times. Here, one can see a substantial difference between the OPV and NQG estimates of imbalance. Generally, the OPV estimate is about an order of magnitude smaller except during the early period of evolution. In this 

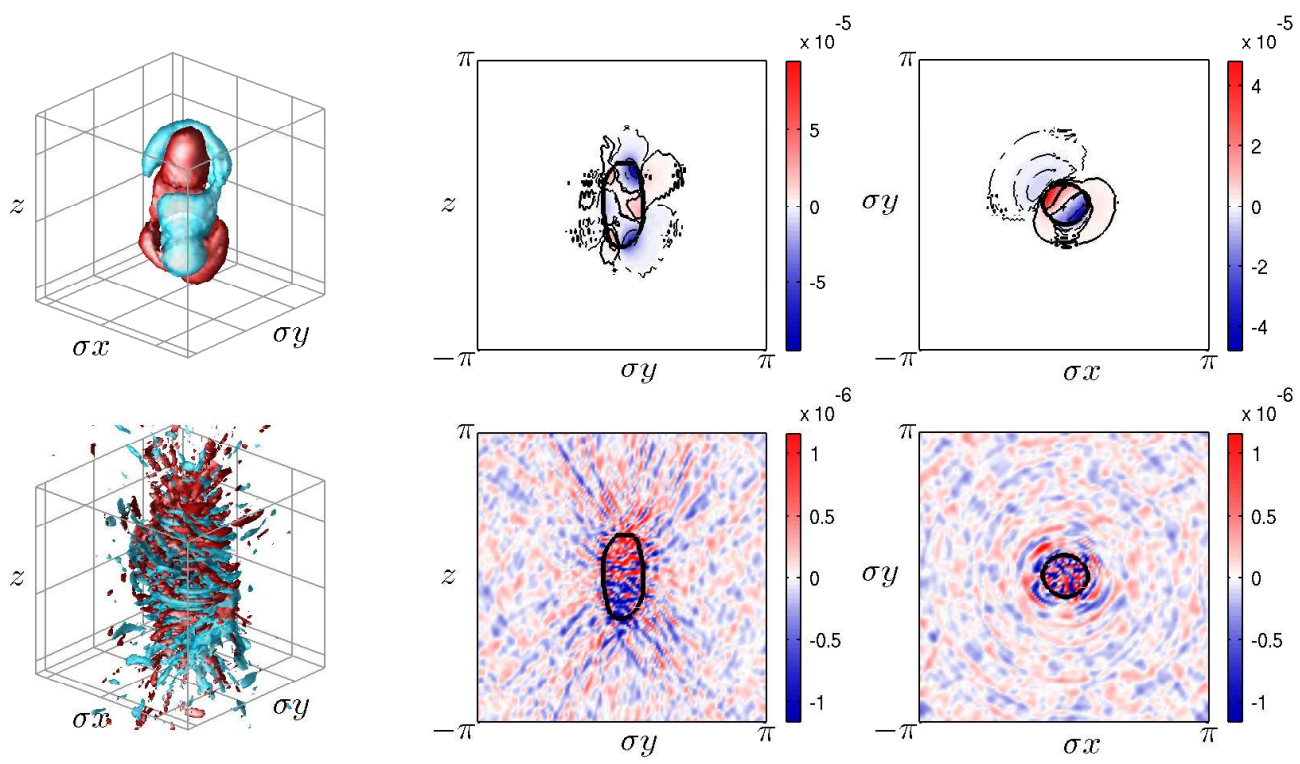

FIGURE 15 . The ageostrophic balanced part $w_{\text {AGb }}$ (top row) and the imbalanced part $w_{\text {imb }}$ (bottom row) of the vertical velocity associated with a cyclonic prolate vortex $\left(q_{0}=0.25, \lambda_{0}=0.7\right.$ and $\left.\mu_{0}=2.4\right)$. The left column shows three-dimensional surfaces at $w_{\mathrm{AGb}}= \pm 1 \times 10^{-5}$ and $w_{\mathrm{imb}}= \pm 6 \times 10^{-7}$ (positive values are in red). The middle column shows a vertical $y$ - $z$ cross section at $x=-\sigma^{-1} \pi / 32$. The right column is a horizontal $x-y$ cross section at $z=-\pi / 32$. A nonlinear color scale is used in the $w_{\text {imb }}$ cross section plots. The maximum $\left|w_{\text {imb }}\right|$ is about $4 \times 10^{-6}$.
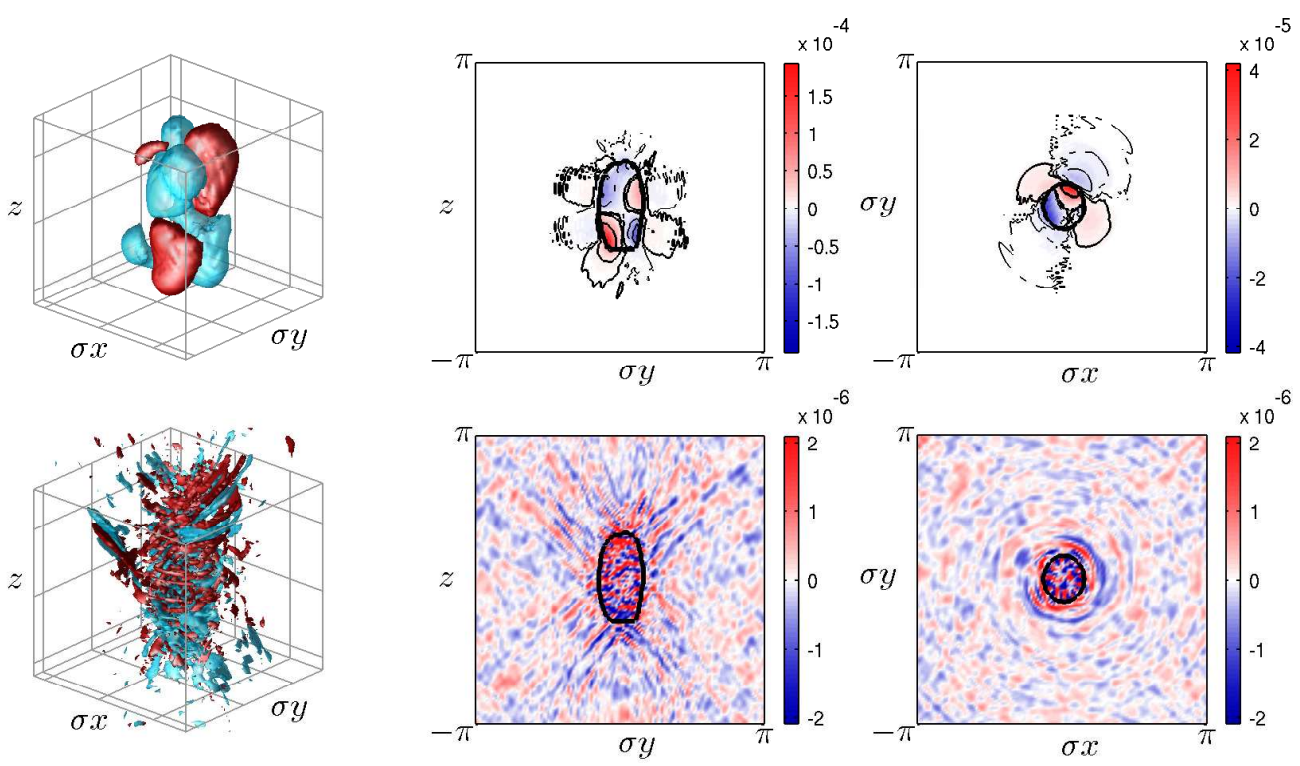

FIGURE 16. As in figure 15 but for an anticyclonic vortex with $q_{0}=-0.25$. Surfaces at $w_{\text {AGb }}= \pm 1 \times 10^{-5}$ and $w_{\text {imb }}= \pm 1.3 \times 10^{-6}$ are shown in the left column. A nonlinear color scale is used in the $w_{\text {imb }}$ cross section plots. The maximum $\left|w_{\text {imb }}\right|$ is about $7 \times 10^{-6}$. 

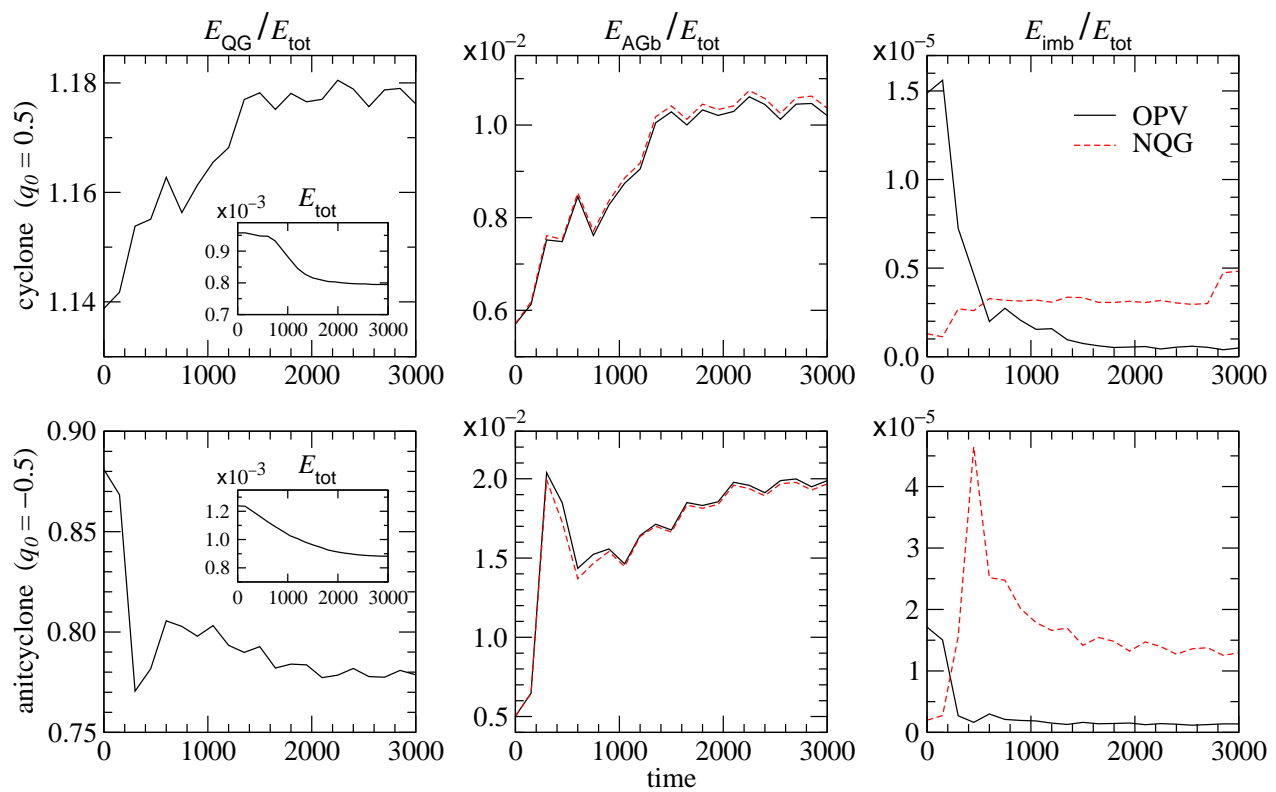

FIGURE 17. Time evolution of the energy norms $E_{\mathrm{QG}}, E_{\mathrm{AGb}}$ and $E_{\mathrm{imb}}$ (defined in (5.4)) for a cyclone and an anticyclone at $q_{0}= \pm 0.5$ respectively. Both vortices have the same initial aspect ratios, $\lambda_{0}=0.2$ and $\mu_{0}=2.4$. The insets show the total energy $E_{\text {tot }}$ versus time.

early period, the flow is rapidly changing due to instability, and OPV struggles to clearly define balance. Indeed, for such time-varying flows, no perfect definition of balance exists; all one can be sure of is that NQG balance provides an overestimate of the imbalance, since presumably one can construct more accurate balance methods for any specific flow situation (cf. Mohebalhojeh \& Dritschel (2001)). OPV balance generally underestimates the imbalance for slowly-evolving flows since inevitably some of the imbalance is attributed to the balanced flow, by construction (see Appendix D). It is less clear what happens for rapidly-evolving flows, but the results of figure 17 indicate that OPV balance overestimates the imbalance in this situation. The upshot is we can bound the actual level of imbalance below the NQG estimate and above the OPV one, provided the OPV estimate is lower than the NQG one.

We next examine how the QG, balanced ageostrophic and imbalanced energies scale with the PV-based Rossby number $q_{0}$. We consider a single vortex shape with $\lambda_{0}=0.3$ and $\mu_{0}=1.6$ for many different values of $q_{0}$, both positive and negative. We compute $E_{\mathrm{QG}}, E_{\mathrm{AGb}}$ and $E_{\mathrm{imb}}$ at or near the final time in each simulation, when the vortex has reached an equilibrium form. The vortex shape at equilibrium depends on $q_{0}$ with $0.68<\langle\lambda\rangle<0.95$ and $1.26<\langle\mu\rangle<1.59$.

In QG theory, the balanced fields are proportional to $q_{0}$, and hence one would expect $E_{\mathrm{QG}}$ to scale with $q_{0}^{2}$. The balanced ageostrophic fields are of higher order, and according to the scaling presented in Appendix $\mathrm{C}$, one would expect $E_{\mathrm{AGb}} \sim q_{0}^{4}$. As for the imbalanced fields, the scaling is less clear. What we find is shown in figure 18 , plotting the values for $E_{\mathrm{QG}}, E_{\mathrm{AGb}}$ and $E_{\mathrm{imb}}$ as a function of $\left|q_{0}\right|$ up to $\left|q_{0}\right|=0.75$, using circles for cyclonic states $\left(q_{0}>0\right)$ and crosses for anticyclonic ones $\left(q_{0}<0\right)$. The QG scaling $E_{\mathrm{QG}} \sim q_{0}^{2}$ is robust at least up to $\left|q_{0}\right|=0.5$, and furthermore $E_{\mathrm{AGb}} \sim q_{0}^{4}$ over all Rossby numbers considered - with consistently higher values for anticyclones (typically 2 to 3 times larger). We find that this is generally true for all the vortex shapes in our principal data set $\left(q_{0}= \pm 0.25, \pm 0.5\right)$, implying that, for the same Rossby number magnitude $\left|q_{0}\right|$, cyclones are closer to QG balance. The imbalance also exhibits a quartic scaling, $E_{\mathrm{imb}} \sim q_{0}^{4}$, albeit with an amplitude $10^{4}$ to $10^{5}$ times smaller than $E_{\mathrm{AGb}}$. There is no clear 


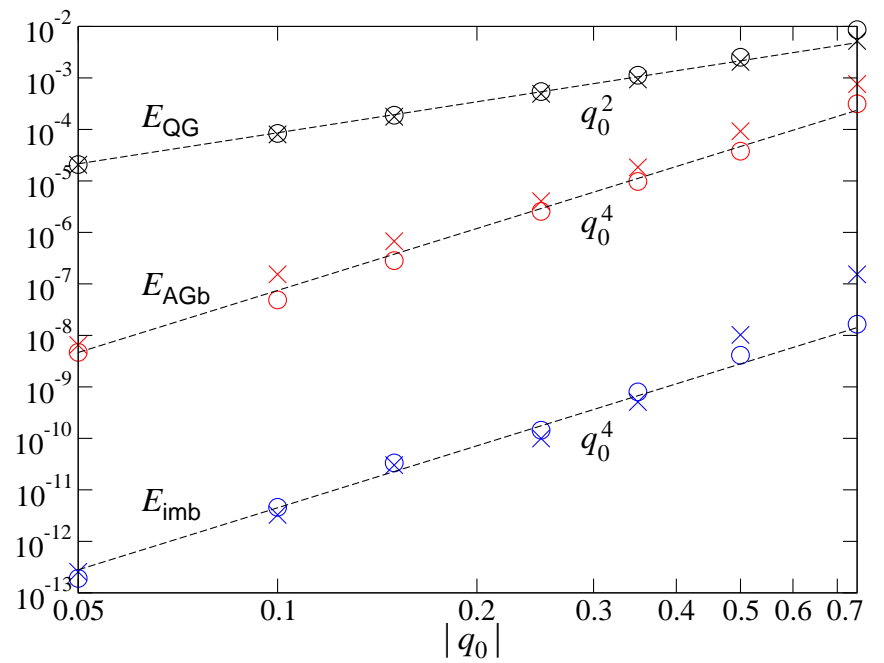

FIGURE 18. Log-log plot of the energy norms for the QG, ageostrophic balanced and imbalanced fields, defined in (5.4), versus the PV-based Rossby number $q_{0}$. Circles represent cyclonic states and crosses anticyclonic states. The initial aspect ratios of the vortex are $\lambda_{0}=0.3$ and $\mu_{0}=1.6$.

trend that anticyclones exhibit more imbalance than cyclones except for $\left|q_{0}\right| \gtrsim 0.5$. However we cannot draw a general conclusion based on just one vortex shape (and it is too costly to examine many different vortex shapes in this way).

\subsection{Discussion}

In the regime $f / N \lesssim 1$, the concept of 'balance', whereby the potential vorticity alone determines all dynamical and thermodynamical fields, has proven immensely useful in understanding geophysical fluid behaviour (Hoskins et al. 1985; Ford et al. 2000; Mohebalhojeh \& Dritschel 2001; McKiver \& Dritschel 2008). Balance arises from filtering, in some way, the inertia-gravity waves from the dynamics. Mathematically, this can be achieved by seeking slowly evolving solutions of the governing equations in which the fluid acceleration $\mathrm{D} \boldsymbol{u} / \mathrm{D} t$ is subdominant. This leads to geostrophic and hydrostatic balance to leading order in the Rossby number, proportional to the ratio of the horizontal acceleration to the Coriolis acceleration.

The quasi-geostrophic (QG) model - describing the advection of a linearised approximation of PV by the leading-order geostrophic velocity field on flat isopycnal surfaces - is the simplest dynamical model imposing balance. However, this model does not distinguish cyclonic and anticyclonic flows (apart from the direction of their rotation). Cyclones and anticyclones are mirror images of each other, dynamically. This mirror symmetry is broken once higher-order effects, arising at second order in Rossby number, are taken into account. In fact, quasi-geostrophic balance does clearly predict this symmetry breaking, i.e. it provides an estimate of (part of) the ageostrophic flow (Charney 1948). It is just that the quasi-geostrophic dynamical model does not make use of this (as elaborated below). Indeed it cannot without a further closure assumption (see Appendix C).

At the QG level of balance, previous well-known results are confirmed, such as increased inertial stability (closer spacing of vortex lines) in cyclones and the opposite in anticyclones, as well as increased static stability $\gamma$ (closer spacing of density surfaces) in cyclones and the opposite in anticyclones. This already hints at the likely increase in stability with the signed Rossby number $q_{0}$, which has been fully confirmed by our results. QG balance, it is worth emphasising, largely explains the difference in stability between cyclones and anticyclones. 
The higher-order ageostrophic balance within the vortex, by contrast, is insignificant. The dominant asymmetry between cyclones and anticyclones is manifest within quasi-geostrophic balance. The reason why this asymmetry does not show up in the QG dynamical model is that it is not used: the QG model advects a linearised approximation of PV on flat isopycnal surfaces using purely geostrophic velocities.

Regarding the residual imbalance, consistent with many previous studies of vortex dynamics and turbulence at PV-based Rossby numbers $<1$, we find that inertia-gravity waves (the imbalance) play a negligible role - at least when the initial conditions are well prepared to contain minimal imbalance. This explains why we can identify 'quasi-equilibria', vortices which persist for long times with very small changes in shape (see Płotka \& Dritschel (2014) for analogous results for shallow-water flows). Essentially, the radiation of inertia-gravity waves is too weak to modify the shape of the vortex over any sensible time period (over time periods extending as far as $10^{3}$ inertial periods ( $\sim$ days) based on the results presented here).

\section{Conclusion}

We have examined one of the most basic problems in geophysical fluid dynamics, namely the behaviour of an isolated vortex in uniform background rotation and stratification. It proves especially convenient to consider a vortex having uniform PV since then one can exploit exact, steadily-rotating solutions having an ellipsoidal shape in the limit of zero Rossby number (vanishingly small PV anomaly relative to the background PV). Properties of these quasi-geostrophic ellipsoids are now well known, including their linear stability and many aspects of their nonlinear behaviour. But the restriction to vanishingly small Rossby number is unrealistic in applications to the Earth's atmosphere and oceans, or to other planetary atmospheres. Our principal aim has been to extend our knowledge of vortex behaviour at finite Rossby number, along the way providing a better understanding of both the limitations and utility of the quasi-geostrophic approximation.

The problem investigated principally depends on three parameters, two describing the shape of the vortex (the horizontal and vertical aspect ratios $\lambda$ and $\mu$ ), and the dimensionless PV anomaly $q_{0}$ within the vortex (the Rossby number). The problem also depends on the Coriolis to buoyancy frequency ratio $f / N$, but only weakly when $f / N \lesssim 1$ (Smyth \& McWilliams 1998; Dritschel $\&$ McKiver 2014). The regime of small $f / N$ is widely applicable to commonly-occurring geophysical flows.

We have examined the stability of vortices covering a wide range of initial shapes $\left(\lambda_{0}, \mu_{0}\right)$ and Rossby numbers $q_{0}$. We have systematically catalogued the wide variety of instabilities which may occur (figure 3), and have identified the quasi-stable equilibrium configurations emerging from these instabilities (figure 4). For these configurations, we have shown that their rotation rate $\Omega$ depends primarily on the late time average value of $\mu$ and $q_{0}$ (figure 6) and that anticyclones rotate faster than cyclones of the same general shape and $\left|q_{0}\right|$. This asymmetry was explained using a higher-order balanced model, and was shown to stem from ageostrophic effects at $\mathcal{O}\left(q_{0}^{2}\right)$.

We have carefully analysed the nature of balance, or the degree to which the PV field alone determines the instantaneous flow. We have shown explicitly that the residual imbalance, characterised by inertia-gravity waves, is negligible across the entire parameter space investigated. The vortex motion depends almost exclusively on the balanced dynamics. Decomposing this into a leading-order, quasi-geostrophic part and a remaining (balanced) ageostrophic part, we have shown that the gross stability properties of the vortices depend primarily on quasi-geostrophic balance, not on the higher-order ageostrophic balance. In other words, the simplest balance quasi-geostrophic balance - is sufficient to explain why cyclones are generally more stable than anticyclones. While this result is not new, our work is the first comprehensive demonstration of it. 
In the present work, we have restricted attention to small frequency ratios $f / N$. A next step would be to study the changes to vortex behaviour that take place for increasing $f / N$, at least up to $\mathcal{O}(1)$ values, keeping the shape and the Rossby number $q_{0}$ of the vortex (or family of vortices) fixed. In rotating-stratified turbulence (Dritschel \& McKiver 2014) however, no significant changes have been found to occur in the basic flow properties for $\left|q_{0}\right| \lesssim 1$ for $f / N$ up to 1 , consistent with the results of Smyth \& McWilliams (1998) who studied the stability of a columnar vortex.

Open questions remain for $\left|q_{0}\right| \gtrsim 1$ that are inaccessible to the approach taken here. Reducing $q_{0}$ much below -0.5 greatly enhances the possibility of inertial instability, and under these circumstances one would expect a violent break-up of the vortex (Smyth \& McWilliams 1998). Increasing $q_{0}$ above 1 would appear to eventually lead to inertial and/or static instability, if one can extrapolate the trends exhibited by the ageostrophic balanced fields in figure 13 and figure 14, for example. As the ageostrophic balanced fields grow quadratically with $q_{0}$ while the QG fields only grow linearly, there may exist a $q_{0}$ sufficiently large when static, inertial or another form of instability occurs (see appendix A.2 in Dritschel \& Viúdez (2003)).

A problem which affects this research and related fundamental studies of vortex dynamics is the present lack of comprehensive observational data. Few ocean vortices, for example, have been analysed in detail (and very little is known about their PV structure), and these few vortices cannot be assumed to be representative. Surface observations alone do not provide vertical structure information, and miss out vortices at depth. Observational analyses are costly and we are not likely to obtain a representative cross section of ocean vortex properties in the near future. What is more promising in the interim is an analysis of ocean vortex properties in high resolution global simulations of the oceans. This would provide much needed support for theoretical studies, and moreover can be done now.

\section{Acknowledgements}

We are indebted to Xavier Carton for his insightful discussions with us. Support for this research has come from the UK Engineering and Physical Sciences Research Council (grant number $\mathrm{EP} / \mathrm{H} 001794 / 1)$.

\section{Appendix A. The equivalent oriented ellipsoid}

Given a volume $\mathcal{V}$ with uniform PV anomaly $q$, we can compute its zeroth, first and second order spatial moments, denoted by $V, \bar{x}_{i}$ and $I_{i j}$ respectively, from the following expressions:

$$
\begin{aligned}
V & =\int_{\mathcal{V}} \mathrm{d} \boldsymbol{x}, \\
\bar{x}_{i} & =\frac{1}{V} \int_{\mathcal{V}} x_{i} \mathrm{~d} \boldsymbol{x}, \\
I_{i j} & =\frac{1}{V} \int_{\mathcal{V}}\left(\tilde{x}_{i} \tilde{x}_{j}-\frac{\delta_{i j}}{3} \sum_{l=1}^{3} \tilde{x}_{l}^{2}\right) \mathrm{d} \boldsymbol{x} .
\end{aligned}
$$

Above, $\boldsymbol{x}=\left(x_{1}, x_{2}, x_{3}\right)=(x, y, z)$ denotes the position vector, $\tilde{\boldsymbol{x}}=\boldsymbol{x}-\overline{\boldsymbol{x}}$ is the position vector relative to the vortex centre at $\overline{\boldsymbol{x}}$, and $\delta_{i j}$ is the Kronecker delta symbol. Note that there are only 9 independent moments in (A 1) since $\sum_{i} I_{i i}=0$.

We define an equivalent oriented ellipsoid $\mathcal{E}$ as one that has the same moments as the vortex up to the second order. This definition slightly differs from the usual one (Chandrasekhar 1987) in that we require the ellipsoid to have the same volume as the vortex. Now, a quadric surface, 
including the ellipsoid, centered at $\boldsymbol{x}^{\prime}$ is represented by the following general equation of second degree,

$$
\left(\boldsymbol{x}-\boldsymbol{x}^{\prime}\right)^{T} \boldsymbol{A}^{-1}\left(\boldsymbol{x}-\boldsymbol{x}^{\prime}\right)=1,
$$

where $\boldsymbol{A}$ is a symmetric $3 \times 3$ matrix. For the equivalent oriented ellipsoid, it is obvious that $\boldsymbol{x}^{\prime}=\overline{\boldsymbol{x}}$. To see the relation between $A_{i j}$ and $I_{i j}$, let $V_{\mathcal{E}}$ be the volume of $\mathcal{E}, a_{i}$ be its semi-axis length in the $x_{i}$-direction and $\boldsymbol{R}$ be the rotation matrix with the property

$$
\boldsymbol{R}^{T} \boldsymbol{A}^{-1} \boldsymbol{R}=\boldsymbol{D}=\left[\begin{array}{ccc}
a_{1}^{-2} & 0 & 0 \\
0 & a_{2}^{-2} & 0 \\
0 & 0 & a_{3}^{-2}
\end{array}\right]
$$

We compute the second moments of $\mathcal{E}$ by transforming $\mathcal{E}$ into the unit sphere $\mathcal{S}$ centred at the origin. This is achieved by the translation $\boldsymbol{x}-\boldsymbol{x}^{\prime}$ followed by the rotation $\boldsymbol{R}$. We finally scale length by $a_{i}$ along the principal axes: $\xi_{k}=a_{k}^{-1} \sum_{i} R_{k i}\left(x_{i}-x_{i}^{\prime}\right)$. Hence,

$$
\begin{aligned}
& \frac{1}{V_{\mathcal{E}}} \int_{\mathcal{E}}\left(x_{i}-x_{i}^{\prime}\right)\left(x_{j}-x_{j}^{\prime}\right) \mathrm{d} \boldsymbol{x} \\
& \quad=\frac{a_{1} a_{2} a_{3}}{V_{\mathcal{E}}} \sum_{k l} R_{i k}\left[\left(a_{k} a_{l}\right) \int_{\mathcal{S}} \xi_{k} \xi_{l} \mathrm{~d} \boldsymbol{\xi}\right] R_{l j}^{T}=\frac{1}{5} \sum_{k l} R_{i k} D_{k l}^{-1} R_{l j}^{T}=\frac{A_{i j}}{5} .
\end{aligned}
$$

Above, the factor $a_{1} a_{2} a_{3}$ is the Jacobian associated with the rotation, and the integral over $\mathcal{S}$ equals $4 \pi \delta_{k l} / 15$. By the definition of equivalent oriented ellipsoid, we thus have,

$$
A_{i j}-\frac{\delta_{i j}}{3} \sum_{l} A_{l l}=5 I_{i j} .
$$

The shape and orientation of the ellipsoid can then be deduced from the eigenvalues and eigenvectors of $\boldsymbol{A}$ (see e.g. McKiver \& Dritschel (2003) for a straightforward derivation).

\section{Appendix B. Estimation of instantaneous vortex rotation rate}

In this appendix, we present a method to estimate the vortex rotation rate from the velocity field at a single time instant. This method is used when the rotation rate, for example $\Omega_{0}$ at $t=0$, cannot be obtained by tracking the rotation of the equivalent ellipsoid. The strategy is to find an optimal co-rotating frame in which the vortex shape appears to be least unsteady, and to identify the angular velocity $\Omega$ of this frame as the vortex rotation rate. The vortex shape is delineated by the PV contours and if a PV contour $\mathcal{C}_{l}$ on an isopycnal surface is steady, the velocity on $\mathcal{C}_{l}$ is tangent to $\mathcal{C}_{l}$. Hence, to represent the unsteadiness, we compute the square of the non-tangential component of the velocity integrated around all contours in a rotating frame and summed over all surfaces:

$$
\mathcal{F}(\Omega)=\sum_{l} \oint_{\mathcal{C}_{l}}\left|\boldsymbol{u}^{\prime}-\left(\boldsymbol{u}^{\prime} \cdot \hat{\boldsymbol{e}}_{t}\right) \hat{\boldsymbol{e}}_{t}\right|^{2} \mathrm{~d} s .
$$

Here $\boldsymbol{u}^{\prime}=\boldsymbol{u}+\Omega \boldsymbol{a}$ (with $\boldsymbol{a} \equiv(y,-x, 0)$ ) is the velocity in the rotating frame and $\hat{\boldsymbol{e}}_{t}$ is the unit tangent vector along $\mathcal{C}_{l}$. We seek $\Omega$ by minimising $\mathcal{F}(\Omega)$; therefore setting $\mathrm{d} \mathcal{F} / \mathrm{d} \Omega=0$ gives

$$
\Omega=-\frac{\sum_{l} \oint_{\mathcal{C}_{l}}\left[\boldsymbol{u} \cdot \boldsymbol{a}-\left(\boldsymbol{u} \cdot \hat{\boldsymbol{e}}_{t}\right)\left(\boldsymbol{a} \cdot \hat{\boldsymbol{e}}_{t}\right)\right] \mathrm{d} s}{\sum_{l} \oint_{\mathcal{C}_{l}}\left[|\boldsymbol{a}|^{2}-\left(\boldsymbol{a} \cdot \hat{\boldsymbol{e}}_{t}\right)^{2}\right] \mathrm{d} s} .
$$


This method works well when the system consists of a single well-defined vortex (which is the case at $t=0$ ). As the vortex evolves in time, filaments and satellites may be spun off, and tracking the equivalent ellipsoid becomes the preferred method.

\section{Appendix C. Nonlinear quasi-geostrophic balance}

Consider a rotating stratified fluid described by (2.10) with the definitions of $\boldsymbol{A}_{\mathrm{h}}$ and $q$ given by (2.9) and (2.16) respectively. Introduce the small parameter $\epsilon \equiv \max |q|$ and recall $\sigma=f / N$. Using the standard QG scalings

$$
t \sim \epsilon^{-1}, \quad(u, v) \sim \epsilon, \quad w \sim \sigma \epsilon^{2}, \quad b \sim \sigma \epsilon
$$

motivates the following expansions of the components of the vector potential $\varphi=(\varphi, \psi, \phi)$ :

$$
\begin{aligned}
\phi & =\epsilon \phi_{1}+\epsilon^{2} \phi_{2}+\mathcal{O}\left(\epsilon^{3}\right), \\
\boldsymbol{\varphi}_{\mathrm{h}} & =\epsilon^{2} \boldsymbol{\varphi}_{\mathrm{h} 2}+\mathcal{O}\left(\epsilon^{3}\right) .
\end{aligned}
$$

In Nonlinear Quasi-Geostrophic (NQG) balance, these expansions are used together with the exact definition of PV in (2.16) to obtain the most accurate second-order estimate of balance known (McKiver \& Dritschel 2008). Substituting (C 2) into (2.16), the QG system appears at order $\epsilon$,

$$
q=\left(\nabla_{\mathrm{h}}^{2}+\sigma^{2} \partial_{z z}\right) \phi_{1}
$$

- except that here $q$ is the exact Rossby-Ertel PV anomaly. At order $\epsilon^{2}$, we obtain a system of linear, constant-coefficient equations for the second-order potentials:

$$
\begin{aligned}
\sigma^{-2}\left(\nabla_{\mathrm{h}}^{2}+\sigma^{2} \partial_{z z}\right) \varphi_{2}+\left(1-\sigma^{-2}\right)\left(\partial_{x x} \varphi_{2}+\partial_{x y} \psi_{2}\right) & =-2 J_{y z}\left(\partial_{x} \phi_{1}, \partial_{y} \phi_{1}\right) \\
\sigma^{-2}\left(\nabla_{\mathrm{h}}^{2}+\sigma^{2} \partial_{z z}\right) \psi_{2}+\left(1-\sigma^{-2}\right)\left(\partial_{x y} \varphi_{2}+\partial_{y y} \psi_{2}\right) & =-2 J_{z x}\left(\partial_{x} \phi_{1}, \partial_{y} \phi_{1}\right) \\
\sigma^{-2}\left(\nabla_{\mathrm{h}}^{2}+\sigma^{2} \partial_{z z}\right) \phi_{2}+\left(1-\sigma^{-2}\right)\left(\partial_{x z} \varphi_{2}+\partial_{y z} \psi_{2}\right) & =0 .
\end{aligned}
$$

Above, $J_{y z}(F, G)=\partial_{y} F \partial_{z} G-\partial_{z} F \partial_{y} G$ with $J_{z x}(F, G)$ analogously defined. The third equation $(\mathrm{C} 4 c)$ in this set incorporates a constraint needed to close the equations at second order, namely that the second-order potentials make no contribution to the PV at order $\epsilon^{2}$. For a given PV anomaly $q$ at any instant of time, the NQG system can be solved for $\phi_{1}$ and $\varphi_{2}$ iteratively. The velocity and the buoyancy fields can then be obtained from (2.13) and (2.14) respectively.

\section{Appendix D. Optimal potential vorticity balance}

For simplicity, consider a given PV field represented by a single contour $C^{*}$ of PV value $q^{*}$. The objective of Optimal Potential Vorticity (OPV) balance is to find a potential $\varphi^{*}(x)$ such that the target balanced flow specified by $\left\{C^{*}, \varphi^{*}\right\}$ has minimum inertia-gravity wave emission. This is achieved, approximately, using the iterative backwards-forwards time integration cycle introduced in Viúdez \& Dritschel (2004). During each cycle, the PV value on the contour changes according to

$$
q(t)=q^{*} \sin ^{2}\left(\frac{\pi t}{2 \tau}\right) .
$$

At $t=0$, the system is in a state of rest. The idea is to very slowly ramp up $q(t)$ while evolving the full set of equations to minimise the emission of IGWs. As a result, the system at $t=\tau$ represents an estimate of the target balanced state.

The cycle starts at $t=\tau$ with the PV contour $C(\tau)=C^{*}$ and some $\varphi(x, \tau)$ which is an estimate of the target potential $\varphi^{*}(x)$. To improve the estimate, we integrate the system $C(t)$ 


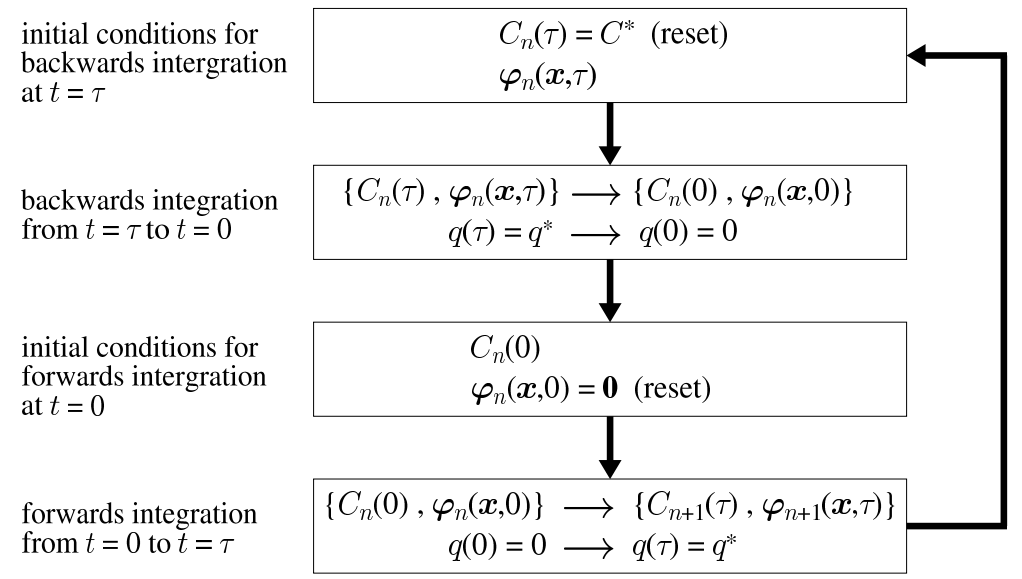

FIGURE 19. Schematic of the OPV balance procedure. At the beginning of the $n$th iteration, the contour $C_{n}$ is reset to $C^{*}$. The system is then integrated backwards in time from $\tau$ to 0 while the PV is ramped down from $q^{*}$ to 0 according to (D 1). Since $q(0)=0, \boldsymbol{\varphi}_{n}(\boldsymbol{x}, 0)$ contains only the imbalance which is removed by setting $\varphi_{n}(\boldsymbol{x}, 0)=0$. Then, a forwards integration from $t=0$ to $t=\tau$ with the PV being ramped up from 0 to $q^{*}$ produces the potential $\varphi_{n+1}(\boldsymbol{x}, \tau)$ for the next iteration. To start the cycle, the 'frozen' initialisation (see text) is used to supply an initial guess $\varphi_{0}(\boldsymbol{x}, \tau)$. The procedure is said to converge when $\left|\boldsymbol{\varphi}_{n}(\boldsymbol{x}, \tau)-\boldsymbol{\varphi}_{n-1}(\boldsymbol{x}, \tau)\right|$ is sufficiently small. We then have the desired potential $\boldsymbol{\varphi}^{*}=\boldsymbol{\varphi}_{n}$.

and $\varphi(\boldsymbol{x}, t)$ backwards in $t$, letting $C(t)$ vary as the flow distorts it. Upon arriving at $t=0$, $q(0)=0$ but we generally find $\varphi(x, 0) \neq 0$. This indicates that we have not yet converged. We reset $\boldsymbol{\varphi}(\boldsymbol{x}, 0)=0$ and integrate forward from a state of rest. Upon arriving at $t=\tau$, we have an improved estimate of $\boldsymbol{\varphi}^{*}(\boldsymbol{x})$ in $\boldsymbol{\varphi}(\boldsymbol{x}, \tau)$ but now $C(\tau)$ will generally not coincide with $C^{*}$. We reset $C(\tau)=C^{*}$ and integrate backwards again, and so on. The cycle ends when the difference between $\varphi(\boldsymbol{x}, \tau)$ at two successive iterations is smaller than some prescribed tolerance.

To start the cycle described above, we need an initial estimate $\varphi(x, \tau)$ in the first iteration. This is obtained as follows. Starting with a state of rest at $t=0$, we 'freeze' $C(t)=C^{*}$ for all $t$ and integrate the remaining equations forward to $t=\tau$ to get a first guess for $\boldsymbol{\varphi}(\boldsymbol{x}, \tau)$.

Figure 19 describes the OPV procedure schematically. As shown in Viúdez \& Dritschel (2004), convergence is generally exponentially fast except when $\tau$ is taken to be too large. Here, we take $\tau=4 T_{\text {ip }}$, which works well in most cases and is consistent with the suggested choice in Viúdez \& Dritschel (2004).

\section{REFERENCES}

CARlson, B. C. \& Gustafson, J. L. 1994 Asymptotic approximations for symmetric elliptic integrals. SIAM J. Math. Anal. 25, 288-303.

CARTon, X. 2001 Hydrodynamical modelling of oceanic vortices. Surveys in Geophysics 22, 179-263.

Carton, X. 2010 Oceanic vortices. Lect. Notes Phys. 805, 61-108.

ChANDRASEKHAR, S. 1987 Ellipsoid Figures of Equilibrium. Dover, New York.

Charney, J. G. 1948 On the scale of atmospheric motions. Geofys. Publ. Oslo 17, 1-17.

Chelton, D. B., Schlax, M. G. \& Samelson, R. M. 2011 Global observations of nonlinear mesoscale eddies. Prog. Oceanogr. 111, 167-216.

Cushman-Roisin, B. 1986 Linear stability of large, elliptical warm-core rings. J. Phys. Oceanogr. 16, $1158-1164$.

Cushman-Roisin, B., HeIL, W. H. \& NOF, D. 1985 Oscillations and rotations of elliptical warm-core rings. J. Geophys. Res. 90, 11756-11764. 
DritsChel, D. G. 1990 The stability of elliptical vortices in an external straining flow. J. Fluid Mech. 210, 223-261.

DritsChel, D. G. \& AmbAUM, M. H. P. 1997 A contour-advective semi-lagrangian numerical algorithm for simulating fine-scale conservative dynamical fields. Q. J. R. Met. Soc. 123, 1097-1130.

DRITSCHEL, D. G. \& MCKIVER, W. J. 2014 Effect of Prandtl's ratio on balance in geophysical turbulence. Undre review.

Dritschel, D. G., SCOTt, R. K. \& Reinaud, J. N. 2005 The stability of quasi-geostrophic ellipsoid vortices. J. Fluid Mech. 536, 401-421.

DritsChel, D. G. \& VIÚDEZ, Á. 2003 A balanced approach to modelling rotating stably stratified geophysical flows. J. Fluid Mech. 488, 123-150.

DRITSCHEL, D. G. \& VIÚDEZ, Á. 2007 The persistence of balance in geophysical flows. J. Fluid Mech. 570, 365-383.

FontANE, J. \& DRITSChel, D. G. 2009 The HyperCASL algorithm: a new approach to the numerical simulation of geophysical flows. J. Comput. Phys. 228, 6411-6425.

Ford, R., MCINTYRE, M. E. \& NORTON, W. A. 2000 Balance and the slow quasimanifold: some explicit results. J. Atmos. Sci. 57, 1236-1254.

HASHIMOto, H., SHIMONISHI, T. \& MiYAZAKI, T. 1999 Quasigeostrophic, ellipsoidal vortices in a twodimensional strain field. J. Phys. Soc. Jpn. 68 (12), 3863-3880.

Hebert, D., OAKEY, N. \& RUdDICK, B. 1990 Evolution of a Mediterranean salt lens: scalar properties. J. Phys. Oceanogr. 20, 1468-1483.

Hoskins, B. J., MCIntyre, M. E. \& Robertson, A. W. 1985 On the use and significance of isentropic potential-vorticity maps. Quart. J. Roy. Meteorol. Soc. 111, 877-946.

HoU, T. Y. \& LI, R. 2007 Computing nearly singular solutions using pseudo-spectral methods. J. Comput. Phys. 226, 379-397.

KIDA, S. 1981 Motion of an elliptic vortex in a uniform shear flow. J. Phys. Soc. Jpn. 50 (10), 3517-3520.

KIM, S. Y. 2010 Observations of submesoscale eddies using high-frequency radar-derived kinematic and dynamic quantities. Continental Shelf Research 30 (15), 1639-1655.

KIRCHHOFF, G. 1876 Vorlesungen über Mathematische Physik: Mechanik. Teubner, Leipzig.

LAMB, S. H. 1932 Hydrodynamics, 6th edn. Cambridge University Press.

Lentini, C. A. D., Goni, G. J. \& Olson, D. B. 2006 Investigation of Brazil Current rings in the confluence region. J. Geophys. Res.: Oceans 111, 2156-2202.

McDowell, S. E. \& Rossby, H. T. 1978 Mediterranean Water: an intense mesoscale eddy off the Bahamas. Science 202, 1085-1087.

MCKiver, W. J. \& DRITSChel, D. G. 2003 The motion of a fluid ellipsoid vortex in a general linear background flow. J. Fluid Mech. 474, 147-173.

McKiver, W. J. \& Dritschel, D. G. 2006 The stability of a quasi-geostrophic ellipsoid vortex in a background strain flow. J. Fluid Mech. 560, 1-17.

MCKiver, W. J. \& Dritschel, D. G. 2008 Balance in non-hydrostatic rotating stratified turbulence. $J$. Fluid Mech. 596, 201-219.

Meacham, S. P. 1992 Quasigeostrophic, ellipsoidal vortices in a stratified flow. Dyn. Atmos. Oceans 16, 189-223.

Meacham, S. P., Flirel, G. R. \& Send, U. 1990 Vortices in shear. Dyn. Atmos. Oceans 14, 333-386.

Meacham, S. P., Morrison, P. J. \& Flirel, G. R. 1997 Hamiltonian moment reduction for describing vortices in shear. Phys. Fluids 9 (8), 2310.

Meacham, S. P., Pankratov, K. K., Shchepetkin, A. F. \& Zhmur, V. V. 1994 The interaction of ellipsoidal vortices with background shear flows in a stratified fluid. Dyn. Atmos. Oceans 21, 167-212.

Meleshro, V. V. \& VAn Heisst, G.J.F. 1994 On Chaplygin's investigations of two-dimensional vortex structures in an inviscid fluid. J. Fluid Mech. 272, 157.

MiyazAKi, T., UenO, K. \& Shimonishi, T. 1999 Quasigeostrophic, tilted spheroidal vortices. J. Phys. Soc. Jpn. 68 (8), 2592-2601.

Mohebalhojeh, A. R. \& Dritschel, D. G. 2001 Hierarchies of balance conditions for the $f$-plane shallow water equations. J. Atmos. Sci. 58(16), 2411-2426.

Moore, D. W. \& Saffman, P. G. 1971 Aircraft Wake Turbulence and Its Detection, p. 339. Plenum Press, New York. 
Muraki, D. J., SNyder, C. \& Rotunno, R. 1999 The next-order corrections to quasigeostrophic theory. J. Atmos. Sci. 56, 1547-1560.

Ólafsdóttir, E. I., Olde DaAlhuis, A. B. \& VAnneste, J. 2008 Inertia-gravity-wave radiation by a sheared vortex. J. Fluid Mech. 596, 169-189.

Olson, D. B. 1991 Rings in the ocean. Annu. Rev. Earth Planet. Sci. 19, 283-311.

Paillet, J., Le Cann, R., Carton, X., Morel, Y. \& Serpette, A. 2002 Dynamics and evolution of a northern meddy. J. Phys. Oceanogr. 32, 55-79.

PARK, K.-A., WoO, H.-J. \& RYU, J.-H. 2012 Spatial scales of mesoscale eddies from GOCI chlorophyll$\alpha$ concentration images in the East/Japan Sea. Ocean Sci. J. 47 (3), 347-358.

PŁotKA, H. \& DRITschel, D. G. 2012 Quasi-geostrophic shallow-water vortex-patch equilibria and their stability. Geophys. Astrophys. Fluid Dyn. 106, 574-595.

PŁOTKA, H. \& DRITSChel, D. G. 2014 Simply-connected vortex-patch shallow-water quasi-equilibria. J. Fluid Mech. 743, 481-502.

Prater, M. D. \& SAnford, T. B. 1994 A meddy off Cape St. Vincent. Part I: Description. J. Phys. Oceanogr. 24, 1572-1586.

Rankine, W. J. M. 1858 A Manual of Applied Mechanics. Griffin, London.

SALMON, R. 1998 Lectures on Geophysical Fluid Dynamics. Oxford University Press, New York.

Smyth, W. D. \& MCWILliams, J. C. 1998 Instability of an axisymmetric vortex in a stably stratified, rotating environment. Theoret. Comput. Fluid Dynamics 11, 305-322.

SPENCE, T. W. \& LEGECKIS, R. 1981 Satellite and hydrographic observations of low-frequency wave motions associated with a cold core Gulf Stream ring. J. Geophys. Res. 86, 1945-1953.

Vallis, G. K. 2006 Atmospheric and Oceanic Fluid Dynamics. Cambridge University Press, Cambridge.

VIÚDEZ, Á. 2007 The origin of the stationary frontal wave packet spontaneously generated in rotating stratified vortex dipoles. J. Fluid Mech. 593, 359-383.

ViúdeZ, Á. \& Dritschel, D. G. 2004 Optimal potential vorticity balance of geophysical flows. J. Fluid Mech. 521, 343-352.

Warn, T., Bokhove, O., Shepherd, T. G. \& VAllis, G. K. 1995 Rossby number expansions, slaving principles, and balance dynamics. Q. J. R. Met. Soc. 121, 723-739.

Young, W. R. 1986 Elliptical vortices in shallow water. J. Fluid Mech. 171, 101-119.

ZhMuR, V. V. \& ShChepetKIN, A. F. 1991 Evolution of an ellipsoidal vortex in a stratified ocean: survivability of the vortex in flow with vertical shear. Izv. Akad. Nauk. SSSR Phys. Atmos. Ocean. 27, 492.

Žunić, J., KopAnJA, L. \& FieldSEnd, J. E. 2006 Notes on shape orientation where the standard method does not work. Pattern Recognition 39, 856-865. 